\title{
Low-coverage surface diffusion in complex periodic energy landscapes. II. Analytical solution for systems with asymmetric hops
}

\author{
Miguel A. Gosálvez, ${ }^{1,2,3, *}$ Mikhail M. Otrokov, ${ }^{2,4,5}$ Nestor Ferrando, ${ }^{2,3}$ Anastasia G. Ryabishchenkova, ${ }^{4}$ Andres Ayuela, ${ }^{2,3}$ \\ Pedro M. Echenique, ${ }^{1,2,3}$ and Evgueni V. Chulkov ${ }^{1,2,3,5}$ \\ ${ }^{1}$ Department of Materials Physics, University of the Basque Country UPV/EHU, 20018 Donostia-San Sebastian, Spain \\ ${ }^{2}$ Donostia International Physics Center (DIPC), 20018 Donostia-San Sebastian, Spain \\ ${ }^{3}$ Centro de Física de Materiales (CFM)-Materials Physics Center (MPC), Centro Mixto CSIC-UPV/EHU, \\ 20018 Donostia-San Sebastian, Spain \\ ${ }^{4}$ Tomsk State University, 634050 Tomsk, Russia \\ ${ }^{5}$ Saint Petersburg State University, 198504 Saint Petersburg, Russia
}

(Received 1 September 2015; revised manuscript received 26 January 2016; published 10 May 2016)

\begin{abstract}
This is part II in a series of two papers that introduce a general expression for the tracer diffusivity in complex, periodic energy landscapes with $M$ distinct hop rates in one-, two-, and three-dimensional diluted systems (low coverage, single-tracer limit). While Part I [Gosálvez et al., Phys. Rev. B 93, 075429 (2016)] focuses on the analysis of diffusion in systems where the end sites of the hops are located symmetrically with respect to the hop origins (symmetric hops), as encountered in many ideal surfaces and bulk materials, this report (Part II) presents a more general approach to determining the tracer diffusivity in systems where the end sites can be located asymmetrically with respect to the hop origins (asymmetric hops), as observed in reconstructed and/or chemically modified surfaces and/or bulk materials. The obtained diffusivity formulas for numerous systems are validated against kinetic Monte Carlo simulations and previously reported analytical expressions based on the continuous-time random walk (CTRW) method. The proposed method corrects some of the CTRW formulas and provides new expressions for difficult cases that have not been solved earlier. This demonstrates the ability of the proposed formalism to describe tracer diffusion.
\end{abstract}

DOI: 10.1103/PhysRevB.93.205416

\section{INTRODUCTION}

The ability of different adsorbates to diffuse quickly or slowly on a given substrate is described by the low coverage diffusion coefficient (or tracer diffusivity [1]),

$$
D_{T}^{\theta \approx 0}=\frac{1}{2 \alpha} \lim _{t \rightarrow \infty} \frac{\left\langle|\mathbf{r}(t)-\mathbf{r}(0)|^{2}\right\rangle}{t},
$$

where $\alpha=1,2,3$ is the number of dimensions, $\mathbf{r}(t)$ designates the position of the diffusing particle (or tracer) at time $t$, and $\langle\cdot\rangle$ is an ensemble average. Although the presentation below is steered towards surface diffusion $(\alpha=2)$, the overall formalism is valid for any $\alpha$.

The traditional approach to determining $D_{T}^{\theta \approx 0}$ using Eq. (1) consists in a labor-intensive averaging procedure where numerous kinetic Monte Carlo (KMC) simulations are performed in order to generate a large collection of random walks outlined by the tracer, whose average reveals the regularities of the propagation in time. Alternatively, tracer diffusion has been previously studied using the continuous-time random walk (CTRW) formalism [2,3], where the diffusivity is obtained in reciprocal space by finding the longest-living eigenvalue of a matrix equation that results from Fourier and Laplace transformations of the master equation. The primary objective of both Part I [1] and Part II of the present study is to provide an alternative, less laborious route to obtaining the diffusivity.

For cross-reference to figures, tables, and equations introduced in Part I of this study [1], this report (Part II) refers to

\footnotetext{
*miguelangel.gosalvez@ehu.es; http://dipc.ehu.es/gosalvez
}

them using the index "PI" in front. For instance, Fig. 1, Table II, and Eq. (3) in Part I are cited here as Fig. PI-1, Table PI-II, and Eq. PI-(3).

Based on the principle of detailed balance, Part I of this study [1] concludes that $D_{T}^{\theta \approx 0}$ can be simply expressed as

$$
D_{T}^{\theta \approx 0}=\frac{1}{2 \alpha} \Sigma_{i=1}^{S} w_{i}\left(\Sigma_{j=1}^{S} \mu_{i j} l_{i j}^{2}\right),
$$

where $S$ is the number of different adsorption sites, $l_{i j}$ is the hop distance from site $i$ to site $j, \mu_{i j}=m_{i j} v_{i j}$ is the rateplicity, defined as the product of the hop rate from site $i$ to site $j$ $\left(v_{i j}\right)$ and its multiplicity $\left(m_{i j}\right)$, which designates the number of equivalent hops from $i$ to $j$, and $w_{i}$ is the occupancy of site $i$ (or probability of residence at site $i$ ), which can be obtained from the rateplicities:

$$
w_{i}=\frac{\frac{\mu_{j i}}{\mu_{i j}}}{\Sigma_{k} \frac{\mu_{j k}}{\mu_{k j}}} \quad(i, j=1, \ldots, S) .
$$

In Eq. (3), any $j$ can be used, although for simplicity we typically use the same $j$ for all $i$ 's.

The benefit of Eq. (2) is that it directly enables calculation of the diffusivity of any adsorbate in a wide variety of complex energy landscapes without performing any simulations of the corresponding random walks. The only necessary input is the values of the hop rates, which are determined from the energy barriers of the hops (diffusion barriers). These are typically obtained from calculations based on density functional theory (DFT). To illustrate the approach, we have estimated the diffusivities of $\mathrm{Se}, \mathrm{Cu}, \mathrm{Ag}$, and $\mathrm{Rb}$ on the (0001) surface of a model topological insulator, $\mathrm{Bi}_{2} \mathrm{Se}_{3}$ [4,5], and discussed the 
diffusion and intercalation of the three latter species in the $\mathrm{Bi}_{2} \mathrm{Se}_{3}$ van der Waals gaps [1].

In addition to simplifying the formulation of the diffusion coefficient according to Eq. (2), the occupancies $\left(w_{i}\right)$ and rateplicities $\left(\mu_{i j}\right)$ also provide a convenient approach to express detailed balance. In the context of diffusion, this principle states that the probability of performing a hop from site type $i$ to site type $j$ is equal to the probability of performing the reverse hop from site type $j$ to site type $i$. As shown in Part I, this can be expressed as

$$
w_{i} \mu_{i j}=w_{j} \mu_{j i} \quad(i, j=1,2, \ldots, S) .
$$

Accordingly, summing over $j$ in Eq. (4) gives the following, less conventional expression for detailed balance:

$$
w_{i} \Sigma_{j=1}^{S} \mu_{i j}=\Sigma_{j=1}^{S} w_{j} \mu_{j i} \quad(i=1,2, \ldots, S),
$$

where the left-hand side can be described as the flux (of particles) out of site $i$, and the right-hand side as the flux into site $i$. Thus, detailed balance requires that the net flux is 0 .

In the given form, Eq. (2) is limited to periodic systems where all the hops are symmetric, meaning that the end sites of the hops are always symmetrically located with respect to the hop origins. Although this behavior is found in a large variety of systems, including many ideal surfaces and bulk materials, there are many examples of periodic systems where the end sites can be located asymmetrically with respect to the hop origins. These asymmetric hops can occur in chemically modified bulk materials and/or reconstructed surfaces (Sec. II A) but also in ideal surfaces and bulk materials (e.g., Table III). Accordingly, this report focuses on the presentation of a more general expression for the tracer diffusivity in such systems. As expected, the generalized expression can be applied directly to the systems featuring symmetric hops only and, thus, is reduced to Eq. (2) in that case.

After a few examples of systems that contain asymmetric hops are presented in Sec. II A, the rest of the paper focuses on the following four major aspects:

(i) The derivation of a generalized expression for the low coverage diffusivity for systems with asymmetric hops and, possibly, boundary crossings (Sec. II). In particular, Sec. II introduces the use of an orthorhombic unit cell (with mutually perpendicular lattice vectors) that enables analysis of the adparticle diffusion along the different Cartesian directions. This leads to the definition of the so-called cell-commensurate sites (whose periodicity is the same as that of the orthorhombic unit cell) as well as the terminal sites (located along the cell boundaries perpendicular to the chosen Cartesian direction). An algorithm is then introduced (method M-1) to assign effective hop rates to the terminal sites, thus describing diffusion as if all meaningful hops would occur between the opposite sides of the cell. For systems with boundary crossings, where the boundaries of the cell are traversed by cell-incommensurate hops, we define the so-called crosser sites, for which the corresponding effective hop rates need to be added to describe the diffusivity. Section II B contains many definitions and may fatigue the reader. However, after their assimilation the description of the diffusivity in Sec. II C becomes elementary. (ii) The derivation of an alternative procedure (method M-2) in order to determine the effective hop rates for the terminal and crosser sites based on a one-dimensional (1D) analysis (Secs. III and IV). In particular, Sec. III applies method M-1 to describe the diffusivity for 1D systems with hops between nearest neighbors in a closed form, and Sec. IV describes the actual procedure for using the derived 1D formulas to determine the effective hop rates for systems in more than one dimension.

(iii) The numerical validation of all the derived formulas for the low coverage diffusivity against KMC simulations of the corresponding random walks, followed by a comparison to previous studies based on the continuous-time random walk formalism (Sec. V) and the conclusions of the study (Sec. VI).

(iv) The derivation of explicit formulas for the low coverage diffusivity for numerous systems (Appendixes 1-4; see [14]). Together with Secs. II E and IIF, Appendix 1 [14] shows that the diffusivity is independent of the choice of unit cell. Appendixes 2 and 3 [14] provide examples for systems with and without boundary crossings, respectively, and Appendix 4 [14] considers additional systems for comparison to previous studies.

\section{LOW COVERAGE DIFFUSIVITY FOR SYSTEMS WITH ASYMMETRIC HOPS}

\section{A. Asymmetric hops}

A hop with origin at site type $i$ and end at site type $j$ is said to be symmetric if site $i$ is surrounded by two or more accessible sites of type $j$ symmetrically located with respect to $i$. In two dimensions, this means that, altogether, the chosen hop and all equivalent hops (with the same origin, length, and rate along other directions) display rotational, $n$-fold symmetry with $n=2,3,4$, and 6 . This corresponds to oppositelinear, trigonal-planar, square-planar, and hexagonal-planar geometries, respectively. In three dimensions, the directions along which the symmetric hops can occur correspond to the tetrahedral, octahedral, cubic, and cuboctahedral geometries ( $n=4,6,8$, and 12, respectively). Otherwise, if one or several end sites are located asymmetrically around the hop origin, the hop is said to be asymmetric.

Figure 1(a) shows a system where one of the hops is asymmetric $\left(v_{21}\right)$. The $v_{12}$ hops have twofold symmetry, and as a result, they propagate the random walker symmetrically towards either side of the $X$ dimension. Similarly, the $v_{23}$ and $v_{32}$ hops also have twofold symmetry, advancing the walker towards both sides of the $Y$ dimension. However, observed from one particular site of type 2, the $v_{21}$ hop (from site 2 to site 1) lacks the opposing hop (also from site 2 to site 1 ), and thus, it contributes to the propagation of the adparticle only towards one side of the $X$ dimension.

Another example is shown in Fig. 1(b). As stressed in inset (i), either of the two $v_{12}$ hops (from site 1 to site 2) lacks the presence of an opposing hop (also from site 1 to site 2). Similarly, inset (ii) emphasizes that the $v_{21}$ hop (from site 2 to site 1) lacks an opposing hop (from site 2 to site 1). In addition, inset (ii) also shows that the $v_{22}^{h}$ hop (in the horizontal direction) and the $v_{22}^{d}$ hops (in the diagonal directions) lack the corresponding opposing hops and, thus, are deemed asymmetric. An additional example is shown in 

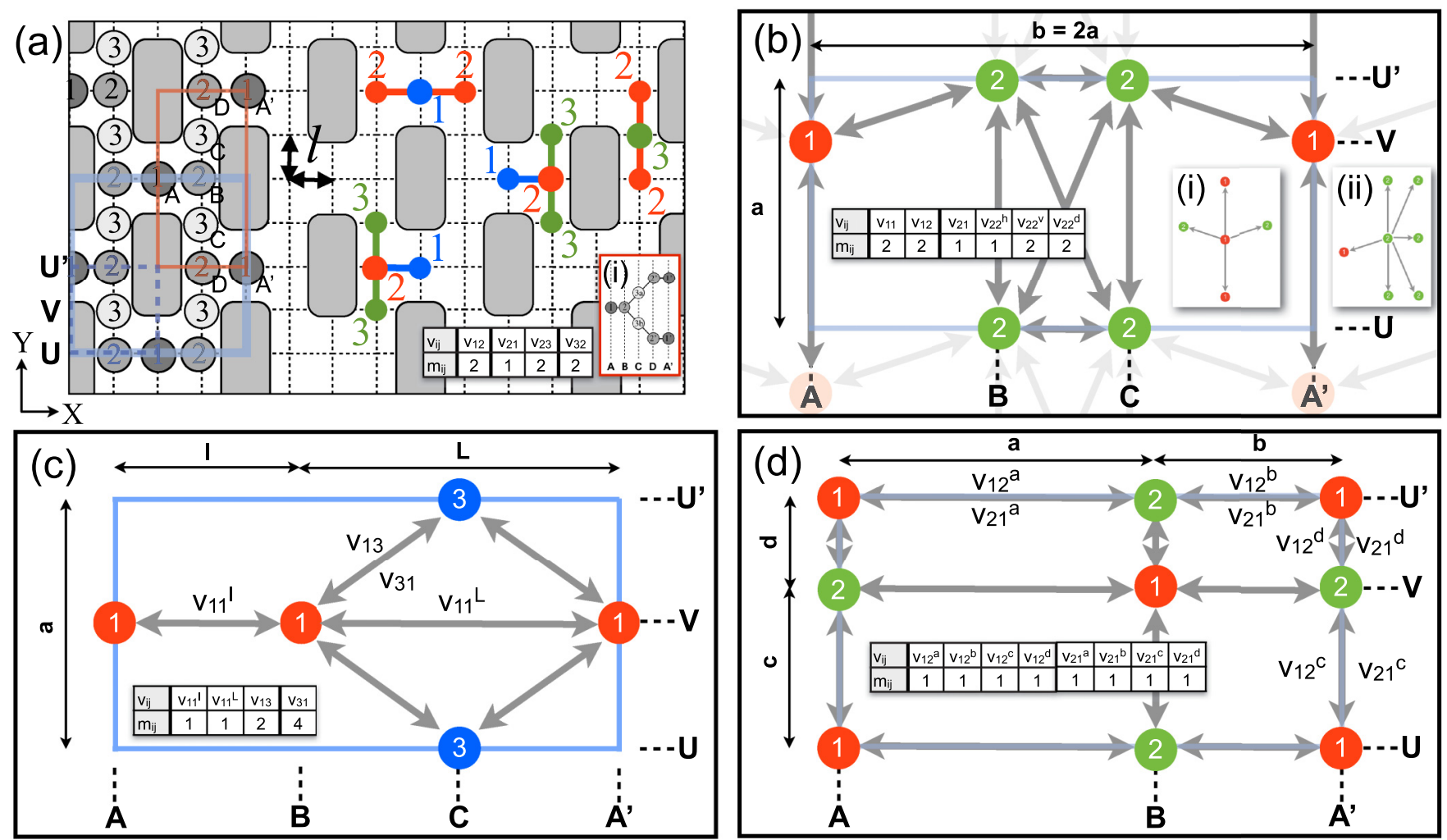

FIG. 1. Example systems with asymmetric hops: (a) Ga adatom on the reconstructed GaAs(001)-c(4×4) surface, based on Ref. [6]. (b) $\mathrm{Ga}$ on the $\mathrm{GaAs}(001)-\mathrm{c}(2 \times 4) \beta 2$ surface, based on case 1 from Ref. [7] (case 2 is considered in Fig. 2$)$. (c) $\mathrm{Cl}$ on the $\mathrm{GaAs}(001)$-c $(8 \times 2)$ $\zeta$ surface, based on Ref. [8]. (d) A general 2D rectangular lattice with two adsorption sites. Insert tables: Considered hop rates and their multiplicities.

Fig. 1(c), where the hops $v_{11}^{l}, v_{11}^{L}$, and $v_{13}$ are asymmetric, while the hops $\nu_{31}$ are symmetric. And a final example is provided in Fig. 1(d), where all the hops are asymmetric.

The previous examples show that the asymmetric hops can be present in varying amounts. Some systems may display a single asymmetric hop among many symmetric ones, while other systems may contain only symmetric or only asymmetric hops. Although hops with a multiplicity larger than 1 can act with both symmetric and asymmetric character, hops with multiplicity 1 always operate asymmetrically. In fact, encountering a hop that has multiplicity 1 is a sufficient condition to conclude that the system contains asymmetric hops. In this manner, if all hop multiplicities in a given system are 2 or larger, then Eq. (2) can be applied as described in Sec. II B of Ref. [1]. However, if at least one multiplicity is 1, then the use of Eq. (2) requires further consideration.

\section{B. Orthorhombic unit cell: Definitions}

The examples in Fig. 1 show that the asymmetric hops introduce asymmetric contributions to the propagation of the diffusing particle. Due to the periodicity of the crystal, however, these asymmetric contributions compensate each other inside the unit cell. As an example, the $\nu_{21}$ hop to the left from site $2_{B}$ to site $1_{A}$ in Fig. 1(a) is compensated by the hop to the right from site $2_{D}$ to site $1_{A^{\prime}}$ inside the displayed unit cell. As a result, the random walker is propagated in a balanced manner about the origin, visiting the positive and negative sides of each dimension with equal probability on average. Thus, the key idea to analyze the diffusivity in systems with asymmetric hops is to define an orthorhombic unit cell [whose lattice vectors are mutually orthogonal and have, possibly, different lengths, as outlined in Figs. 1(a)-1(d)] so that the propagation of the random walker can be split into its Cartesian components (along the corresponding lattice directions) and an overall hop rate across the unit cell can be defined for each dimension. If needed, different unit cells can be used to analyze the propagation along each dimension.

To illustrate the approach, we consider a rather complex system, as shown in Fig. 2(a). The periodicity is accounted for by using an orthorhombic unit cell aligned with the $X$ and $Y$ axes, with $a_{1}=b=2 a$ and $a_{2}=a$. The four displayed adsorption sites (1-4) and six transition states $\left(T_{1}, T_{2}, T_{3}\right.$ and $\left.T_{5}, T_{6}, T_{7}\right)$ correspond to Ga adatoms on the $\mathrm{GaAs}(001)$ $\mathrm{c}(2 \times 4) \beta 2$ surface [7]. With broken lines Fig. 2(a) shows the paths for different hops, which are summarized in the four included tables, one for each site type as the origin of the hops, as represented schematically in Figs. 2(b) and 2(c). The $v_{12} / v_{21}$ and $v_{22}^{d}$ hops highlighted as dark-gray double-headed arrows in Figs. 2(b) and 2(c) correspond to the emphasized (thicker) broken lines in Fig. 2(a).

\section{Relabeling}

Once the unit cell has been chosen it is convenient to relabel all equivalent sites, as shown in Fig. 2(d). Equivalent sites along the chosen dimension ( $X$, in this example) are 
(a)

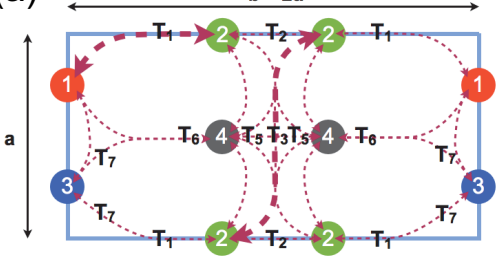

$\mathrm{A}_{1}=-2.5 \quad \mathrm{~A}_{2}=-2.2 \quad \mathrm{~A}_{3}=-3.2 \quad \mathrm{~A}_{4}=-2.6 \quad$ (adsorption energies in $\mathrm{eV}$ ) $\mathrm{T}_{1}=-1.7 \quad \mathrm{~T}_{2}=-1.8 \quad \mathrm{~T}_{3}=-1.6 \quad \mathrm{~T}_{4}=-2.0 \quad \mathrm{~T}_{5}=-1.45 \quad \mathrm{~T}_{6}=-1.5 \quad \mathrm{~T}_{7}=-1$. (b)

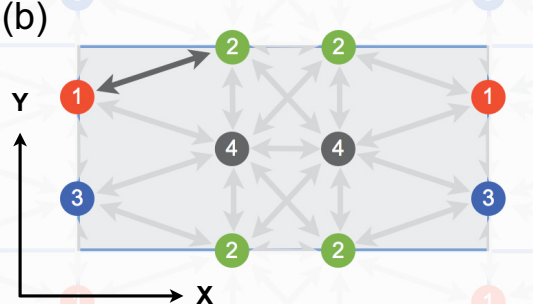

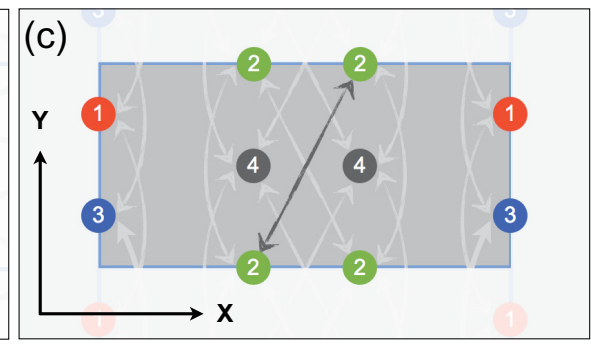

$\mathrm{h}=$ horizontal; $\mathrm{v}=$ vertical; $\mathrm{d}=$ diagonal

\begin{tabular}{|c|c|c|c|c|c|c|c|c|c|c|c|c|c|c|c|c|c|c|c|c|c|c|}
\hline $\mathrm{v}_{\mathrm{ii}}$ & $v_{11}$ & $v_{12}$ & $v_{13}$ & $v_{14}$ & $v_{21}$ & $\mathrm{v}_{22^{\mathrm{h}}}$ & $\mathrm{v}_{22^{\mathrm{v}}}$ & $\mathrm{v}_{22^{\mathrm{d}}}$ & $v_{23}$ & $v_{24}{ }^{v}$ & $\mathrm{~V}_{24^{\mathrm{d}}}$ & $\mathrm{v}_{31}$ & $\mathrm{v}_{32}$ & $\mathrm{v}_{33}$ & $\mathrm{v}_{34}$ & $v_{41}$ & $\mathrm{v}_{42^{\mathrm{v}}}$ & $\mathrm{V}_{42^{\mathrm{d}}}$ & $\mathrm{V}_{43}$ & $\mathrm{~V}_{44^{\mathrm{h}}}$ & $\mathrm{V}_{44^{\mathrm{V}}}$ & $\mathrm{V}_{44^{\mathrm{d}}}$ \\
\hline$m_{i j}$ & 2 & 2 & 2 & 2 & 1 & 1 & 2 & 2 & 1 & 2 & 2 & 2 & 2 & 2 & 2 & 1 & 2 & 2 & 1 & 1 & 2 & 2 \\
\hline$E_{a}=\ldots$ & $\mathrm{T}_{7}-\mathrm{A}_{1}$ & $T_{1}-A_{1}$ & $\mathrm{~T}_{7}-\mathrm{A}_{1}$ & $\mathrm{~T}_{6}-\mathrm{A}_{1}$ & $T_{1}-A_{2}$ & $\mathrm{~T}_{2}-\mathrm{A}_{2}$ & $\mathrm{~T}_{3}-\mathrm{A}_{2}$ & $\mathrm{~T}_{3}-\mathrm{A}_{2}$ & $\mathrm{~T}_{1}-\mathrm{A}_{2}$ & $\mathrm{~T}_{5}-\mathrm{A}_{2}$ & $\mathrm{~T}_{5}-\mathrm{A}_{2}$ & $T_{7}-A_{3}$ & $\mathrm{~T}_{1}-\mathrm{A}_{3}$ & $\mathrm{~T}_{7}-\mathrm{A}_{3}$ & $\mathrm{~T}_{6}-\mathrm{A}_{3}$ & $\mathrm{~T}_{6}-\mathrm{A}_{4}$ & $T_{5}-A_{4}$ & $T_{5}-A_{4}$ & $\mathrm{~T}_{6}-\mathrm{A}_{4}$ & $\mathrm{~T}_{5}-\mathrm{A}_{4}$ & $\mathrm{~T}_{5}-\mathrm{A}_{5}$ & $\mathrm{~T}_{5}-\mathrm{A}_{5}$ \\
\hline$E_{a}(\mathrm{eV})$ & 0.6 & 0.8 & 0.6 & 1.0 & 0.5 & 0.4 & 0.6 & 0.6 & 0.5 & 0.75 & 0.75 & 1.3 & 1.5 & 1.3 & 1.7 & 1.1 & 1.15 & 1.15 & 1.1 & 1.15 & 1.15 & 1.15 \\
\hline
\end{tabular}
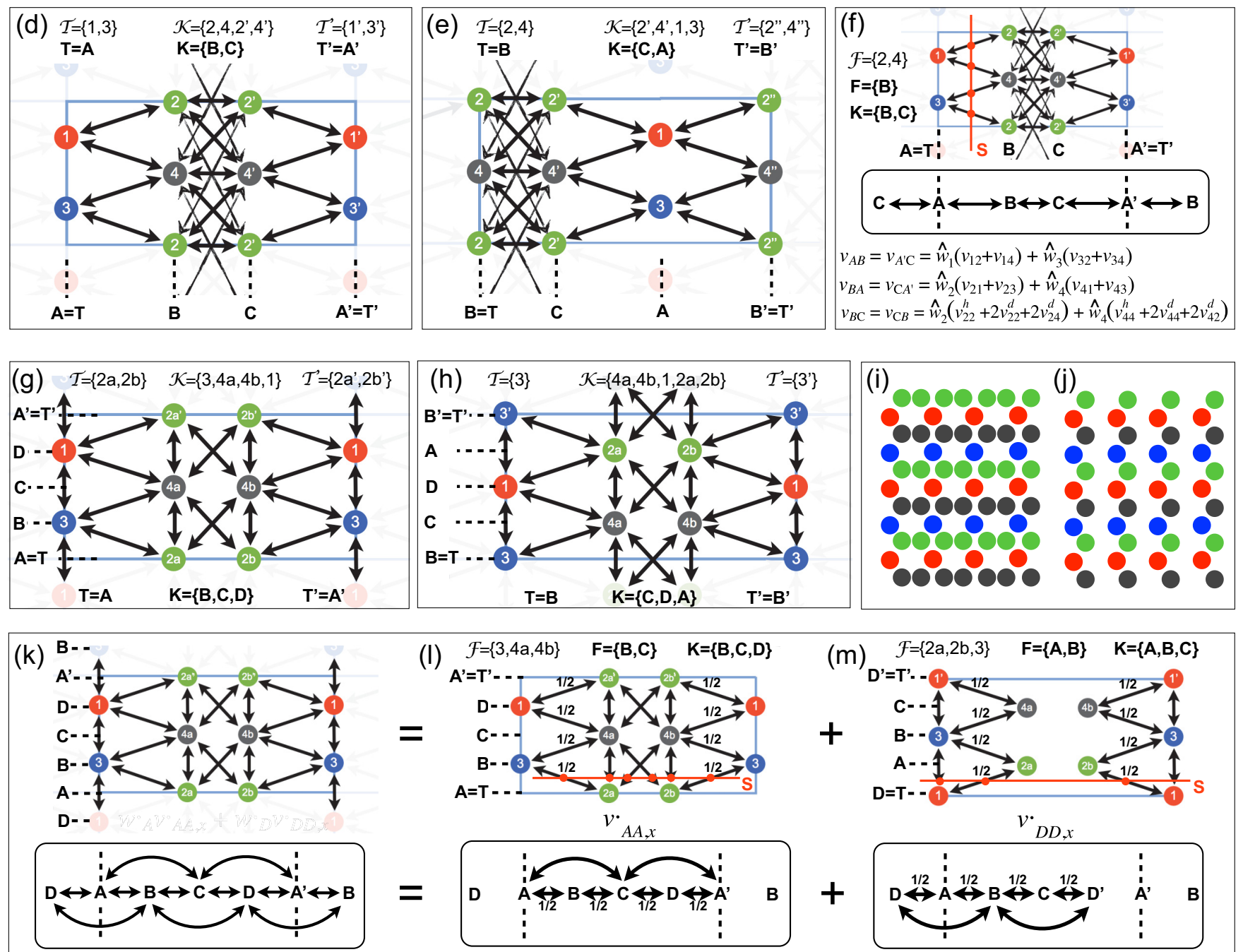

Examples of hop rates between planes: $\quad v_{A B}=\hat{w}_{2 a} v_{23}+\hat{w}_{2 b} v_{23}=v_{23} \quad v_{A \mathrm{C}}=v_{A^{\prime} \mathrm{C}}=\hat{w}_{2 a}\left(v_{24}^{v}+v_{24}^{d}\right)+\hat{w}_{2 b}\left(v_{24}^{v}+v_{24}^{d}\right)=v_{24}^{v}+v_{24}^{d} \quad v_{B \mathrm{C}}=\hat{w}_{3} 2 v_{34}=2 v_{34}$

FIG. 2. Prototypical system with asymmetric hops: Ga on the GaAs(001)-c(2×4) $\beta 2$ surface, based on case 2 in Ref. [7]. (a) Location of the four adsorption sites $\left(A_{1}-A_{4}\right.$ or, simply, 1-4) and six transition states $\left(T_{1}-T_{3}\right.$ and $\left.T_{5}-T_{7}\right)$, showing the corresponding energies (in eV) and the list of all defined hop rates. Hops highlighted in light and dark gray are represented graphically in (b) and (c), respectively. (d) and (e) Two alternative unit cells to study transport along the $X$ dimension. (f) Equivalent 1D representation of transport along $X$ for the unit cell in (d). Hop rates between planes are shown. (g) and (h) Two alternative unit cells to study transport along $Y$. (i) and (j) The probability of finding a particle located at a green or black site in (i) is double that in (j). (k) Equivalent 1D representation of transport along $Y$ for the unit cell in (g). Some hop rates between planes are given (last line of text). (l) and (m) Decomposition of the hops in (k) between the primary cell (l) and the complementary cell $(\mathrm{m})$ in order to determine the effective hop rates $v_{\mathbf{A A}, x}^{*}$ and $v_{\mathbf{D D}, x}^{*}$. 
distinguished using primed symbols, such as $2,2^{\prime}, 2^{\prime \prime}, 2^{\prime \prime \prime} \ldots$ Equivalent sites along a perpendicular dimension $(Y$, in this example) are relabeled using alphabetical letters, such as $2 \mathrm{a}$, $2 b, 2 c$..., with the exception of those sites located at the cell boundaries parallel to the chosen dimension, which should not be relabeled. Additional relabeling examples are shown in Figs. 2(e)-2(g). Note that the multiplicities of the hops are affected by this relabeling, and thus, the rateplicities as well.

After relabeling, the effective occupancy of site $i$ is

$$
w_{i}^{*}=\frac{w_{i}}{n_{i}},
$$

where $n_{i}$ is the number of times that site $i$ appears in the chosen unit cell before relabeling. As an example, for Fig. 2(d) we have $w_{1}^{*}=w_{1}, w_{2}^{*}=w_{2^{\prime}}^{*}=w_{2} / 2, w_{3}^{*}=w_{3}$, and $w_{4}^{*}=w_{4^{\prime}}^{*}=$ $w_{4} / 2$. We recall that the occupancy is the fraction of time that the particle spends at a site or probability of residence (see Part I of this study [1]). Thus, $w_{2}$ is the probability of being found at site 2 or at site $2^{\prime}$. Accordingly, the probability of being found at site 2 is $w_{2}^{*}=w_{2} / 2$ and the probability of being found at site $2^{\prime}$ is $w_{2^{\prime}}^{*}=w_{2} / 2$. Figures 2(i) and 2(j) show this visually. For Fig. 2(g) we have $w_{1}^{*}=w_{1}, w_{2 a}^{*}=w_{2 b}^{*}=w_{2} / 2$, $w_{3}^{*}=w_{3}$, and $w_{4 a}^{*}=w_{4 b}^{*}=w_{4} / 2$.

\section{Cell-commensurate, terminal, and inner sites}

A hop is said to be cell commensurate along dimension $l$ if the projection of the hop distance along dimension $l$ is equal to the length $a_{l}$ of the unit cell. For instance, hops $v_{11}, v_{22}^{v}$ and $v_{22}^{d}$ in Fig. 1(b) are cell commensurate along $Y$. The set $\mathcal{C}$ is defined as the collection of cell-commensurate sites, which are the origins (and destinations) of the cell-commensurate hops. Thus, sites 1 and 2 in Fig. 1(b) belong to $\mathcal{C}$. As a rule, these hops have multiplicity 2 , since they take place in both the forward and the backward directions, where forward (backward) means towards the positive (negative) end of the chosen dimension. According to Fig. 2(c), the hops $v_{11}, v_{33}, v_{22}^{v}, v_{22}^{d}, v_{44}^{v}$, and $v_{44}^{d}$ are cell commensurate along $Y$.

We also define other types of sites. This includes the lower and upper terminal sites, located at the lower and upper boundaries of the unit cell, i.e., the cell terminals perpendicular to the chosen dimension $l$. Here, lower (upper) means towards the negative (positive) end of the chosen dimension. Correspondingly, the set $\mathcal{T}$ is defined as the collection of lower boundary sites (lower terminal). Similarly, $\mathcal{T}^{\prime}$ is the set of upper boundary sites (upper terminal). In addition, we define $\mathcal{K}$ (for "kernel") as the set of inner sites, located between the two boundaries. For Fig. 2(d) we have $\mathcal{T}=\{1,3\}, \mathcal{T}^{\prime}=\left\{1^{\prime}, 3^{\prime}\right\}$, and $\mathcal{K}=\left\{2,4,2^{\prime}, 4^{\prime}\right\}$. Other examples are shown in Figs. 2(e)-2(h).

\section{Effective hop rate for sites}

The idea behind the definition of the terminal sites is that all internal hops inside the unit cell can be replaced by a few effective hops between the terminal sites. In this manner, we define $v_{i i, l}^{*}$ to be the effective hop rate from terminal site $i$ to terminal site $i$ for dimension $l$. This hop rate has multiplicity 2 , since the hops take place in the forward and backward directions. As an example, in Fig. 2(d) (propagation along $X$ ), the terminal sites are 1 and 3 , and thus we need to determine $v_{11, x}^{*}$ and $v_{33, x}^{*}$. Alternatively, we could choose the unit cell shown in Fig. 2(e), with terminal sites 2 and 4, thus requiring the determination of $v_{22, x}^{*}$ and $v_{44, x}^{*}$. However, the final expression for the diffusivity does not depend on the choice of unit cell, and thus, we may focus on either case. Similarly, for the effective hop rates along Y $\left(v_{i i, y}^{*}\right)$, Fig. $2(\mathrm{~g})$ shows a possible choice for the unit cell, with $2 a$ and $2 b$ as the terminal sites, leading to the use of $v_{2 a 2 a, y}^{*}$ and $v_{2 b 2 b, y}^{*}$. An alternative would be the unit cell shown in Fig. 2(h), where the terminal site is 3 , thus leading to $v_{33, y}^{*}$.

In addition, we define $v_{i i^{\prime}, l}^{+}\left(v_{i^{\prime} i, l}^{-}\right)$as the forward (backward) effective hop rate along direction l for terminal site $i\left(i^{\prime}\right) . v_{i i^{\prime}, l}^{+}$ is the rate to hop forward from site $i$ in the lower terminal $\mathcal{T}$ to the equivalent site $i^{\prime}$ in the upper terminal $\mathcal{T}^{\prime}$. Similarly, $v_{i^{\prime} i, l}^{-}$ is the rate to hop backward from site $i^{\prime}$ in the upper terminal $\mathcal{T}^{\prime}$ to the equivalent site $i$ in the lower terminal $\mathcal{T}$. The forward and backward rates must be equal. In fact, their value defines the effective hop rate for site $i: v_{i i, l}^{*}=v_{i i^{\prime}, l}^{+}=v_{i^{\prime} i, l}^{-}$.

\section{One-dimensional representation}

We also define a set of planes $\mathbf{A}, \mathbf{B}, \mathbf{C}, \ldots, \mathbf{X}, \mathbf{A}^{\prime}$ perpendicular to the propagation direction, such that every plane contains at least one site type of the relabeled system. As an example, the planes $\mathbf{A}, \mathbf{B}, \mathbf{C}$, and $\mathbf{A}^{\prime}$ are shown in Fig. 2(d) and $\mathbf{A}, \mathbf{B}$, $\mathbf{C}, \mathbf{D}$, and $\mathbf{A}^{\prime}$ are displayed in Fig. 2(g). The planes have the same periodicity as the unit cell. We define $\mathbf{K}=\{\mathbf{B}, \mathbf{C}, \ldots, \mathbf{X}\}$ as the set of inner planes, which contain the inner sites $k \in$ $\mathcal{K}$. For instance, $\mathbf{K}=\{\mathbf{B}, \mathbf{C}\}$ for Fig. $2(\mathrm{~d})$ and $\mathbf{K}=\{\mathbf{B}, \mathbf{C}, \mathbf{D}\}$ for Fig. 2(g). In turn, the lower and upper terminal planes are designated $\mathbf{T}$ and $\mathbf{T}^{\prime}$, respectively. In both Fig. 2(d) and Fig. 2(g) we have $\mathbf{T}=\mathbf{A}$ and $\mathbf{T}^{\prime}=\mathbf{A}^{\prime}$. In both Fig. 2(e) and Fig. 2(h) we have $\mathbf{T}=\mathbf{B}$ and $\mathbf{T}^{\prime}=\mathbf{B}^{\prime}$.

The effective occupancy of plane $\mathbf{P}$ is defined as the sum of the effective occupancies of the sites within $\mathbf{P}$ :

$$
w_{\mathbf{P}}^{*}=\Sigma_{i \in \mathbf{P}} w_{i}^{*} .
$$

It denotes the fraction of time that the adparticle spends at the sites within that plane, i.e., the probability of residence at that plane. As an example, $w_{\mathbf{A}}^{*}=w_{1}^{*}+w_{3}^{*}, w_{\mathbf{B}}^{*}=w_{2}^{*}+w_{4}^{*}, w_{\mathbf{C}}^{*}=$ $w_{2^{\prime}}^{*}+w_{4^{\prime}}^{*}$, and $w_{\mathbf{A}^{\prime}}^{*}=w_{1^{\prime}}^{*}+w_{3^{\prime}}^{*}$ for Fig. 2(d). Similarly, $w_{\mathbf{A}}^{*}=$ $w_{2 a}^{*}+w_{2 b}^{*}, w_{\mathbf{B}}^{*}=w_{3}^{*}, w_{\mathbf{C}}^{*}=w_{4 a}^{*}+w_{4 b}^{*}, w_{\mathbf{D}}^{*}=w_{1}^{*}$, and $w_{\mathbf{A}^{\prime}}^{*}=$ $w_{2 a^{\prime}}^{*}+w_{2 b^{\prime}}^{*}$ for Fig. $2(\mathrm{~g})$. In addition, the in-plane occupancy of site $i$ is defined as

$$
\hat{w}_{i}=\frac{w_{i}^{*}}{w_{\mathbf{P}}^{*}}=\frac{w_{i}^{*}}{\Sigma_{j \in \mathbf{P}} w_{j}^{*}} .
$$

It describes the probability of residence at site $i$ normalized by the total residence probability at the corresponding plane. $\hat{w}_{i}$ is an important quantity, as shown below.

For any two planes $\mathbf{P}$ and $\mathbf{Q}$ (not necessarily contiguous), the hop rate from $\mathbf{P}$ to $\mathbf{Q}\left(\nu_{\mathbf{P Q}}\right)$ is defined as

$$
\begin{gathered}
\nu_{\mathbf{P Q}}=\Sigma_{i \in \mathbf{P}} \hat{w}_{i} \Sigma_{j \in \mathbf{Q}} \mu_{i j}, \\
w_{\mathbf{P}}^{*} \nu_{\mathbf{P Q}}=\Sigma_{i \in \mathbf{P}} w_{i}^{*} \Sigma_{j \in \mathbf{Q}} \mu_{i j} .
\end{gathered}
$$

Equation (9) contains the rates (through the rateplicities $\mu_{i j}$ ) for all the hops from any site within plane $\mathbf{P}$ to any directly connected site within plane $\mathbf{Q}$. Every rateplicity is multiplied by the corresponding in-plane occupancy $\left(\hat{w}_{i}\right)$. Figure 2(f) provides a few examples of this definition in practice. Note 
that, through this definition, the problem of diffusion is reduced to a $1 \mathrm{D}$ analysis. As an example, the propagation along $X$ in the 2D system shown in Fig. 2(f) can be replaced with the 1D system displayed right below it. The same applies to the system shown in Fig. 2(k) for propagation along $Y$. Note also that the rates of the hops perpendicular to the direction of propagation can be completely disregarded, e.g., we may set them to 0. For instance, Figs. 2(d)-2(f) (for propagation along $X$ ) do not use/display any hop along the $Y$ direction. Similarly, Figs. 2(g)-2(m) (for propagation along $Y$ ) do not use/display any hop along the $X$ dimension. We stress that, given a propagation direction, the $1 \mathrm{D}$ representation becomes an essential tool.

\section{Detailed balance for the inner planes}

The flux of particles out of (into) plane $\mathbf{P}$ is defined as

$$
J_{\mathbf{P}}^{\text {out }}=w_{\mathbf{P}}^{*} \Sigma_{\mathbf{Q} \neq \mathbf{P}} v_{\mathbf{P Q}}, \quad J_{\mathbf{P}}^{\text {in }}=\Sigma_{\mathbf{Q} \neq \mathbf{P}} w_{\mathbf{Q}}^{*} v_{\mathbf{Q P}} .
$$

Due to detailed balance the net flux $\left(J_{\mathbf{P}}^{\text {net }}=J_{\mathbf{P}}^{\text {out }}-J_{\mathbf{P}}^{\text {in }}\right)$ must be 0 ,

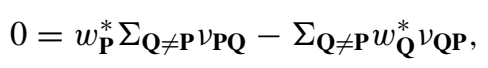

which, for any inner plane $(\mathbf{P} \in \mathbf{K})$, can be rewritten as

$$
w_{\mathbf{P}}^{*} R_{\mathbf{P}}^{\neq}-\Sigma_{\mathbf{Q} \in \mathbf{K}}^{\mathbf{Q} \neq \mathbf{P}} w_{\mathbf{Q}}^{*} v_{\mathbf{Q P}}=w_{\mathbf{T}}^{*} \nu_{\mathbf{T P}}+w_{\mathbf{T}^{\prime}}^{*} v_{\mathbf{T}^{\prime} \mathbf{P}}
$$

where $R_{\mathbf{P}}^{\neq}=\Sigma_{\mathbf{Q} \neq \mathbf{P} \nu_{\mathbf{P Q}}}$ is the total rate out of plane $\mathbf{P}$. Equation (13) is a useful statement of detailed balance for any inner plane $\mathbf{P} \in \mathbf{K}$ and is used repeatedly below.

\section{Effective hop rate for planes}

As for the terminal sites, we define $v_{\mathbf{T T}^{\prime}, l}^{+}\left(\nu_{\mathbf{T}^{\prime} \mathbf{T}, l}^{-}\right)$to be the forward (backward) effective hop rate along direction $l$ for plane $\mathbf{T}\left(\mathbf{T}^{\prime}\right)$. The forward and backward rates must be equal, thus defining the effective hop rate for plane $\mathbf{T}: v_{\mathbf{T T}, l}^{*}=$ $v_{\mathbf{T T}^{\prime}, l}^{+}=v_{\mathbf{T}^{\prime} \mathbf{T}, l}^{-}$. Note that the multiplicity of $v_{\mathbf{T T}, l}^{*}$ is also 2 . Based on Eq. (9), we can express the effective hop rate for the plane in terms of the effective hop rates for the sites:

$$
\begin{gathered}
v_{\mathbf{T T}, l}^{*}=\sum_{i \in \mathcal{T}} \hat{w}_{i} v_{i i, l}^{*}=\frac{1}{w_{\mathbf{T}}^{*}} \sum_{i \in \mathcal{T}} w_{i}^{*} v_{i i, l}^{*}, \\
w_{\mathbf{T}}^{*} v_{\mathbf{T T}, l}^{*}=\sum_{i \in \mathcal{T}} w_{i}^{*} v_{i i, l}^{*}
\end{gathered}
$$

\section{Boundary crossings}

Let us consider the terminal plane $\mathbf{T}$ of a given unit cell. We define $\mathcal{X}_{\text {tot }} \cup \mathcal{B}_{\text {tot }}$ as the total collection of boundary crossing sites, which contains all origin and destination sites for the hops that cross $\mathbf{T}$ and are not cell commensurate, if they exist. $\mathcal{X}_{\text {tot }}$ contains the lower crossers (located from $\mathbf{T}$ towards the negative end of the propagation direction) and $\mathcal{B}_{\text {tot }}$ includes the upper crossers. Accordingly, $\mathbf{X}_{\text {tot }}$ is defined to hold all the lower crosser planes, and $\mathbf{B}_{\text {tot }}$, all the upper crosser planes.

As an example, in Fig. 2(g) (propagation along $Y$ ) the terminal planes $\mathbf{A}$ and $\mathbf{A}^{\prime}$ are crossed by the hops between site 1 and site 3 , located at planes $\mathbf{D}$ and $\mathbf{B}$, respectively. Thus, $\mathcal{X}_{\text {tot }}=$ $\{1\}, \mathcal{B}_{\text {tot }}=\{3\}, \mathbf{X}_{\text {tot }}=\{\mathbf{D}\}$, and $\mathbf{B}_{\text {tot }}=\{\mathbf{B}\}$. Similarly, for Fig. 2(h) we have $\mathcal{X}_{\text {tot }}=\{2 a, 2 b\}, \mathcal{B}_{\text {tot }}=\{4 a, 4 b\}, \mathbf{X}_{\text {tot }}=\{\mathbf{A}\}$, and $\mathbf{B}_{\text {tot }}=\{\mathbf{C}\}$. For Fig. 2(d) (propagation along $X$ ), we have $\mathcal{X}_{\text {tot }}=\mathcal{B}_{\text {tot }}=\mathbf{X}_{\text {tot }}=\mathbf{B}_{\text {tot }}=\varnothing$ (empty). For an example with two upper crosser planes, we may consider the system shown in Fig. 3(a). If we place the lower terminal of the cell at site 2 (plane B), then we would have $\mathbf{B}_{\text {tot }}=\{\mathbf{C}, \mathbf{D}\}$ (with $\mathbf{X}_{\text {tot }}=\{\mathbf{A}\}$ ).

\section{Primary and complementary unit cells}

If boundary crossings take place we refer to the chosen unit cell as the primary cell and define the so-called upper and lower complementary unit cells. Given the planes $\mathbf{A}, \mathbf{B}, \mathbf{C}, \ldots$, $\mathbf{X}, \mathbf{A}^{\prime}$, the upper complementary cell is the result of shifting the perimeter of the primary cell from $\mathbf{A}$ to $\mathbf{B}$. For the unit cell shown in Fig. 2(g), this means the unit cell shown in Fig. 2(h). Similarly, the lower complementary cell is defined by shifting the perimeter from $\mathbf{A}$ to $\mathbf{X}$. For the cell in Fig. $2(\mathrm{~g})$, the result is the cell shown in Fig. 2(m). Note that, by construction, the primary cell can be considered the upper complementary cell of the lower cell. Similarly, the primary cell can also be viewed as the lower complementary cell of the upper cell. Thus, the roles of the primary and complementary cells can be exchanged. They are equally important. For convenience, however, we simply assign them one role or the other. The diffusivity itself does not depend on the choice of the primary cell or the selection of the corresponding complementary cell (see Secs. IIE and IIF). Without loss of generality, for a given primary cell we focus below on the use of the lower complementary cell.

When boundary crossings occur the adparticle can penetrate into the primary cell by a different route than just visiting the terminal sites. Thus, the boundary crossers make a contribution to the diffusivity. In practice, a convenient way to determine this contribution is by considering the (lower) complementary cell and assigning its terminal plane $(\mathbf{X})$ and sites $(i \in \mathcal{X})$ the corresponding effective hop rates $\left(v_{\mathbf{X X}, l}^{*}\right.$ and $v_{i i, l}^{*}$, respectively). As for the previous cases, the multiplicities of $v_{\mathbf{X X}, l}^{*}$ and $v_{i i, l}^{*}$ are 2 , since the hops will take place in both the forward and the backward directions. As in Eq. (15), we can express the sum of the effective hop rates for the sites in terms of the effective hop rate for the plane:

$$
w_{\mathbf{X}}^{*} v_{\mathbf{X} \mathbf{X}, l}^{*}=\sum_{i \in \mathcal{X}} w_{i}^{*} v_{i i, l}^{*} .
$$

\section{Hop-commensurate paths}

Based on the 1D representation, where the hops occur between planes, we define a forward path as a series of $c \geqslant 1$ concatenated hops in the forward direction, with the longest path having the length of the unit cell. Similar definitions are valid for the backward direction. Examples of paths for Fig. 2(f) are $\mathbf{A} \mathbf{B C A} \mathbf{A}^{\prime}$ and $\mathbf{B C A} \mathbf{A}^{\prime}$. In Sec. III we derive expressions for the effective hop rate of such paths. For instance, it is shown that $v_{\mathbf{A B C}}=v_{\mathbf{A B}} v_{\mathbf{B C}} /\left(v_{\mathbf{B A}}+v_{\mathbf{B C}}\right)$. Now, considering Fig. 2(k), we note that some paths provide parallel routes to longer hops. We say that the path and the hop are commensurate. For instance, the path $\mathbf{A B C}$ is commensurate to the hop AC. In general, for a long hop forwards with rate $v_{\mathbf{A L}}$ and a commensurate path with rate $v_{\mathbf{A B} . . \mathbf{L}}$, the effective hop rate $v_{\mathbf{A L}}^{+}$is simply their sum:

$$
v_{\mathbf{A L}}^{+}=v_{\mathbf{A L}}+v_{\mathbf{A B} \ldots \mathbf{L} .} .
$$



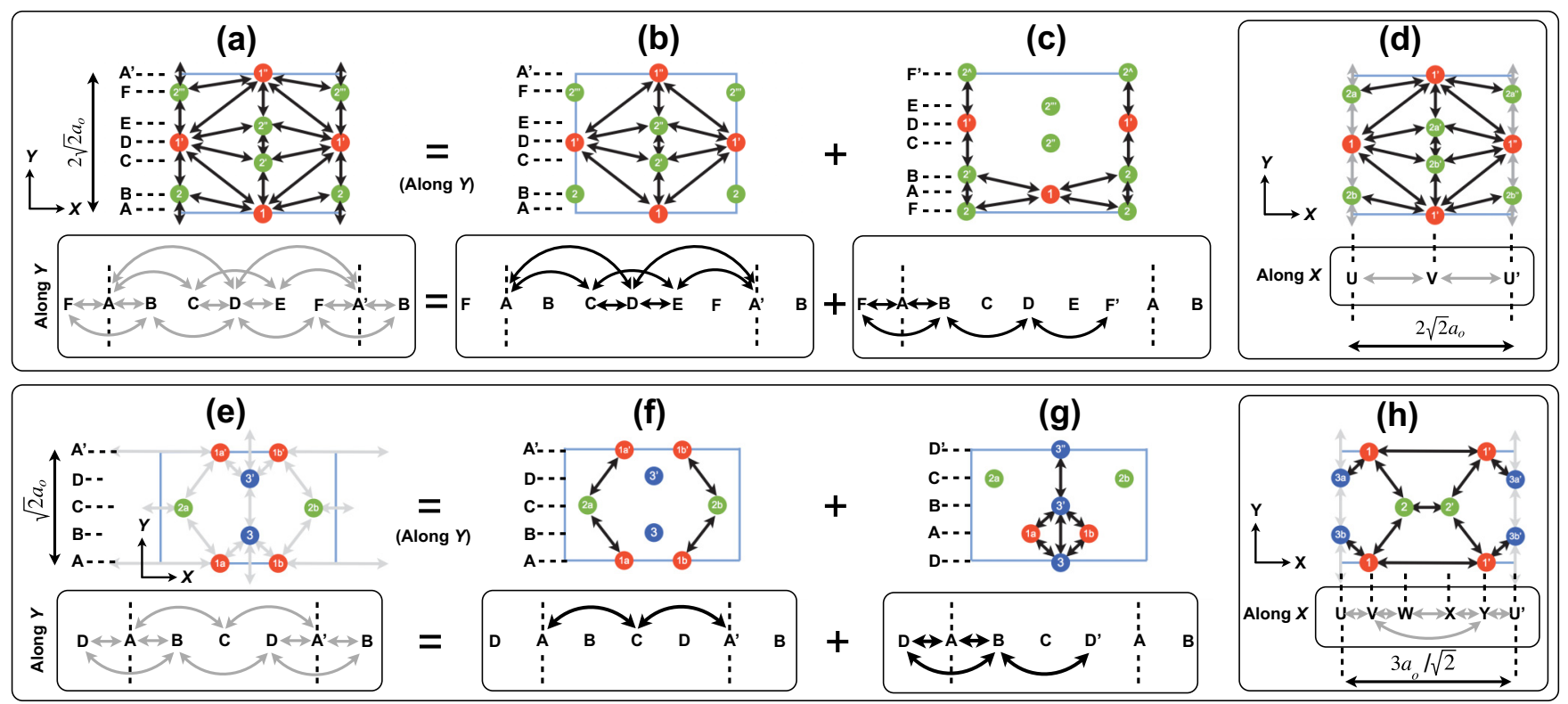

FIG. 3. Additional examples of systems with boundary crossings: (a) Selected unit cell (and 1D representation) for transport along $Y$ for the system studied by Penev et al. [9] (case 4; low temperature). [Case 3, high temperature, is considered in Fig. 5(b).] (b) and (c) Primary and complementary unit cells (and 1D representations) for the system in (a), after distribution of the hops (see text). (d) Same as (a), but for transport along $X$ (no boundary crossings). (e) Same as (a), but for a different study by Penev et al. [10] [case 5; reconstruction (2×3)]. [Case 6, reconstruction $(1 \times 3)$, is considered in Fig. 5(c).] (f) and (g) Same as (b) and (c), but for the system in (e). (h) Same as (e), but for transport along $X$ (no boundary crossings).

This seemingly trivial statement has important consequences, as shown next (Sec. II B 10).

\section{Distributing the hops between the cells}

When boundary crossings occur the determination of $v_{\text {TT }, l}^{*}$ (for the primary cell) and $v_{\mathbf{X X}, l}^{*}$ (for the complementary cell) is done by considering the $1 \mathrm{D}$ representation (where the hops are between planes) and distributing the hops between the two cells according to three rules.

R1: Neither cell may contain hops across its terminals.

R2: Any hop-commensurate path must remain within the same cell as the corresponding long hop.

R3: The combination of the two cells must contain every hop once: no more and no less.

In particular, condition $\mathrm{R} 1$ is ensured by setting to 0 the rates of the hops between the site sets $\mathcal{X}_{\text {tot }}$ and $\mathcal{B}_{\text {tot }}$. Typically, this involves

R1a: zeroing the boundary crossings, i.e., canceling the hops across the lower and upper boundaries of the cell.

R1b: zeroing similar hops elsewhere, if those hops occur between the sites in $\mathcal{X}_{\text {tot }}$ and $\mathcal{B}_{\text {tot }}$. An example is shown in Fig. 2(1) for the primary cell, where $v_{\mathbf{B D}}^{*}=v_{\mathbf{D B}}^{*}=0$. Note that, in the $1 \mathrm{D}$ representation shown in Fig. 2(k), the central hop between plane $\mathbf{B}$ (site 3 ) and plane $\mathbf{D}$ (site 1) has been canceled in Fig. 2(1), even if that hop does not cross the cell terminals. The reason for this is that site 1 is in set $\mathcal{X}_{\text {tot }}$ and site 3 , in $\mathcal{B}_{\text {tot }}$. A similar reasoning is applied to the complementary cell in Fig. 2(m), now with $v_{\mathbf{A C}}^{*}=v_{\mathbf{C A}^{\prime}}^{*}=v_{\mathbf{A}^{\prime} \mathbf{C}}^{*}=v_{\mathbf{C A}}^{*}=0$. Note that this "zeroing elsewhere" does not affect similar hops between sites that formerly were equivalent to sites in $\mathcal{X}_{\text {tot }}$ and $\mathcal{B}_{\text {tot }}$ before relabeling, but are not equivalent afterwards. This is shown in Fig. 3(a), where the hops between planes $\mathbf{F}$ and $\mathbf{B}$ are zeroed out in Fig. 3(b) but the equivalent hop between planes $\mathbf{C}$ and $\mathbf{E}$ remains.

R1c: zeroing any resulting disconnected hops, after the application of R1a and R1b. An example is shown in Fig. 3(c) for the complementary cell, where $v_{\mathbf{C D}}, v_{\mathbf{C E}}$, and $v_{\mathbf{D E}}$ (and the reverse rates) have been zeroed out. These hops cannot contribute to diffusion along $Y$.

The decomposition of the system shown in Fig. 2(k) into those shown in Figs. 2(1) and 2(m) satisfies condition R2 by keeping every hop-commensurate path in the same cell as the corresponding long hop. Note that, when combining the two cells, the hops within the commensurate paths would be counted twice, and thus, we need to divide their rates by 2 in order to satisfy condition R3. In this manner, the figures show a factor of $1 / 2$ at every affected rate. Similarly, the decompositions shown in Figs. 3(a)-3(c) and 3(e)-3(g) have been carried out taking into account conditions R1-R3.

Any other decomposition of the hops between the two cells disregarding conditions R1-R3 will result in an error. For instance, if the commensurate path $\mathbf{A B C}$ is completely removed from Fig. 2(1) [and fully considered in Fig. 2(m)], then the effective rate [Eq. (17)] between planes $\mathbf{A}$ and $\mathbf{C}$ in Fig. 2(1) will be $v_{\mathbf{A C}}^{+}=v_{\mathbf{A C}}$, which wrongly disregards the fact that the adparticle may propagate along the parallel route $\mathbf{A B C}$ in addition to the direct hop AC.

\section{Expressing the diffusivity in terms of the effective hop rates}

After the previous definitions we are now in a position to describe the low coverage tracer diffusivity $\left(D_{T}^{\theta \approx 0}\right)$ in terms of the effective hop rates. For this purpose, the diffusivity is written out in terms of its Cartesian components 
$\left(D_{T, x}^{\theta \approx 0}, D_{T, y}^{\theta \approx 0}, \ldots\right)$ as

$$
D_{T}^{\theta \approx 0}=\frac{1}{\alpha} \Sigma_{l=1}^{\alpha} D_{T, x_{l}}^{\theta \approx 0},
$$

where $\alpha$ is the dimensionality and $D_{T, x_{l}}^{\theta \approx 0}$ is the $l$ th Cartesian component $\left(l=1, \ldots, \alpha ; x_{1}=x, x_{2}=y, x_{3}=z\right)$ :

$$
\begin{aligned}
D_{T, x_{l}}^{\theta \approx 0} & =\frac{1}{2} \lim _{t \rightarrow \infty} \frac{\left\langle\left(x_{l}(t)-x_{l}(0)\right)^{2}\right\rangle}{t} \\
& =\frac{1}{2}\left(\sum_{i \in \mathcal{C}} w_{i} \mu_{i i}+\sum_{i \in \mathcal{T}} w_{i}^{*} \mu_{i i, l}^{*}+\sum_{i \in \mathcal{X}} w_{i}^{*} \mu_{i i, l}^{*}\right) a_{l}^{2} \\
& =\left(\sum_{i \in \mathcal{C}} w_{i} v_{i i}+\sum_{i \in \mathcal{T}} w_{i}^{*} v_{i i, l}^{*}+\sum_{i \in \mathcal{X}} w_{i}^{*} v_{i i, l}^{*}\right) a_{l}^{2} \\
& =\left(\sum_{i \in \mathcal{C} \cup \mathcal{T} \cup \mathcal{X}} w_{i}^{*} v_{i i, l}^{*}\right) a_{l}^{2} .
\end{aligned}
$$

Here, Eq. (1) has been used to go from Eq. (18) to Eq. (19). Similarly, Eq. (2) has been used to write out Eq. (20), expressing $D_{T, x_{l}}^{\theta \approx 0}$ as the sum of three terms. The first term, $\frac{1}{2}\left(\Sigma_{i \in \mathcal{C}} w_{i} \mu_{i i}\right) a_{l}^{2}$, considers the contribution to the diffusivity from the cell-commensurate sites, $i \in \mathcal{C}$, with occupancies $w_{i}$ and rateplicities $\mu_{i i}=2 v_{i i}$. The value of the multiplicity, 2 , is used to go from Eq. (20) to Eq. (21). The second term, $\frac{1}{2}\left(\Sigma_{i \in \mathcal{T}} w_{i}^{*} \mu_{i, l}^{*}\right) a_{l}^{2}$, contains the contribution from the terminal sites, $i \in \mathcal{T}$. The formula uses the effective occupancies $w_{i}^{*}$ to deal correctly with those cases where several equivalents of the terminal sites may appear inside the unit cell. Since the hops between the terminals have multiplicity 2 , the rateplicities are $\mu_{i i, l}^{*}=2 v_{i i, l}^{*}$. The third term, $\frac{1}{2}\left(\Sigma_{i \in \mathcal{X}} w_{i}^{*} \mu_{i i, l}^{*}\right) a_{l}^{2}$, adds the contribution from the boundary crossers, $i \in \mathcal{X}$, with the effective occupancies $w_{i}^{*}$ and rateplicities $\mu_{i i, l}^{*}=2 v_{i i, l}^{*}$. Note that $w_{i}^{*}=w_{i}$ and $v_{i i, l}^{*}=v_{i i}$ for the cell-commensurate sites. For the terminal and crosser sites, $w_{i}^{*}=w_{i} / n_{i}$ (as defined in Sec II B 1) while $v_{i i, l}^{*}$ needs to be determined, as described in Sec. II D below.

Replacing Eq. (22) into Eq. (18), the diffusivity becomes

$$
\begin{aligned}
D_{T}^{\theta \approx 0} & =\frac{1}{2 \alpha} \Sigma_{l}^{\alpha}\left(\sum_{i \in \mathcal{C} \cup \mathcal{T} \cup \mathcal{X}} w_{i}^{*} \mu_{i i, l}^{*}\right) a_{l}^{2} \\
& =\frac{1}{\alpha} \Sigma_{l=1}^{\alpha}\left(\sum_{i \in \mathcal{C} \cup \mathcal{T} \cup \mathcal{X}} w_{i}^{*} v_{i i, l}^{*}\right) a_{l}^{2} \\
& =\frac{1}{\alpha} \Sigma_{l=1}^{\alpha} v_{l} a_{l}^{2},
\end{aligned}
$$

where $v_{l}$ is defined as the overall rate across the unit cell along dimension l:

$$
\begin{aligned}
v_{l} & =\sum_{i \in \mathcal{C} \cup \mathcal{T} \cup \mathcal{X}} w_{i}^{*} v_{i i, l}^{*} \\
& =\sum_{i \in \mathcal{C}} w_{i} v_{i i}+\sum_{i \in \mathcal{T}} w_{i}^{*} v_{i i, l}^{*}+\sum_{i \in \mathcal{X}} w_{i}^{*} v_{i i, l}^{*} .
\end{aligned}
$$

Equation (25) is the main result of this study for systems with asymmetric hops. The diffusivity is the mean of the Cartesian components $v_{l} a_{l}^{2}$ for an orthorhombic unit cell with lattice parameters $a_{l}$, the overall rate $v_{l}$ along dimension $l$ being an occupancy-weighted sum over the effective rates $v_{i i, l}^{*}$ [Eq. (27)]. The form in Eq. (23) strongly resembles Eq. (2) for systems with only symmetric hops, the main difference being that we can directly use the atomistic hop rates and distances in the symmetric case, while we need to determine the effective hop rates and distances for a suitable orthogonal unit cell in the asymmetric case. Both cases use the occupancies $w_{i}$ (or the effective occupancies $w_{i}^{*}=w_{i} / n_{i}$ ), obtained using Eq. (3).

Based on the 1D representation [see Figs. 2(f) and 2(k)], the Cartesian components of the diffusivity are obtained most directly by using the effective hop rates for the terminal and crosser planes [Eqs. (15) and (16)]:

$$
D_{T, x_{l}}^{\theta \approx 0}=\left(\sum_{i \in \mathcal{C}} w_{i} v_{i i}+w_{\mathbf{T}}^{*} v_{\mathbf{T T}, l}^{*}+w_{\mathbf{X}}^{*} v_{\mathbf{X} \mathbf{X}, l}^{*}\right) a_{l}^{2} .
$$

In practice, Eq. (28) provides a fundamental workhorse and is routinely used in this study.

\section{General expression for the effective hop rate}

The previous section has shown that, if boundary crossings occur, the original unit cell can be considered as the combination of a primary and a complementary cell, for which no boundary crossings take place. For each cell, the effective hop rate is calculated following the same general procedure, which is described here. Particular examples of this general procedure are provided in Secs. IIE and II F.

For a unit cell without boundary crossings for propagation direction $l$, we consider the special plane $\mathbf{S}$ located between the lower terminal plane $\mathbf{T}$ and the next plane in the positive propagation direction. Examples are shown in Figs. 2(f), 2(1), and $2(\mathrm{~m})$ for propagation along $X$ and $Y$, respectively. We define $\mathcal{F}$ as the collection of sites that serve as the destination of the hops crossing $\mathbf{S}$. For instance, $\mathcal{F}=\{2,4\}$ for Fig. 2(f), $\mathcal{F}=\{3,4 a, 4 b\}$ for Fig. 2(1), and $\mathcal{F}=\{2 a, 2 b, 3\}$ for Fig. $2(\mathrm{~m})$. Also, we define $\mathbf{F}$ as the set of destination planes, e.g., $\mathbf{F}=$ $\{\mathbf{B}\}$ for Fig. 2(f), $\mathbf{F}=\{\mathbf{B}, \mathbf{C}\}$ for Fig. 2(1), and $\mathbf{F}=\{\mathbf{A}, \mathbf{B}\}$ for Fig. 2(m).

Now the net flux through $\mathbf{S}$ along $l$ must be 0 due to detailed balance:

$$
0=J_{\mathbf{S}}^{l+}-J_{\mathbf{S}}^{l-}=w_{\mathbf{T}}^{*} \Sigma_{\mathbf{P} \in \mathbf{F}} \nu_{\mathbf{T P}}-\Sigma_{\mathbf{P} \in \mathbf{F}} w_{\mathbf{P}}^{*} \nu_{\mathbf{P T}} .
$$

However, from the point of view of the forward and backward effective hop rates, $v_{\mathbf{T T}^{\prime}, l}^{+}$and $\nu_{\mathbf{T}^{\prime} \mathbf{T}, l}^{-}$, the hops occur between the cell terminals, and thus, the only hops crossing plane $\mathbf{S}$ are the effective hops themselves. Correspondingly, the net flux can also be written as

$$
0=w_{\mathbf{T}}^{*} \nu_{\mathbf{T T}^{\prime}, l}^{+}-w_{\mathbf{T}^{\prime}}^{*} \nu_{\mathbf{T}^{\prime} \mathbf{T}, l}^{-} .
$$

Comparison of Eqs. (29) and (30) suggests that expressing the effective occupancy of the destination planes in Eq. (29) ( $w_{\mathbf{P}}^{*}$ with $\mathbf{P} \in \mathbf{F}$ ) in terms of the occupancies of the upper and lower terminal planes in Eq. (30) $\left(w_{\mathbf{T}}^{*}\right.$ and $\left.w_{\mathbf{T}^{\prime}}^{*}\right)$ will enable identification of the forward and backward effective hop rates of Eq. (30) $\left(v_{\mathbf{T T}^{\prime}, x}^{+}\right.$and $\left.\nu_{\mathbf{T}^{\prime} \mathbf{T}, x}^{-}\right)$with corresponding expressions in Eq. (29). To do this, let us consider Eq. (13) (detailed balance) for all inner planes $\mathbf{P} \in \mathbf{K}=\{\mathbf{B}, \mathbf{C}, \ldots, \mathbf{X}\}$, which, in 
matrix form, gives

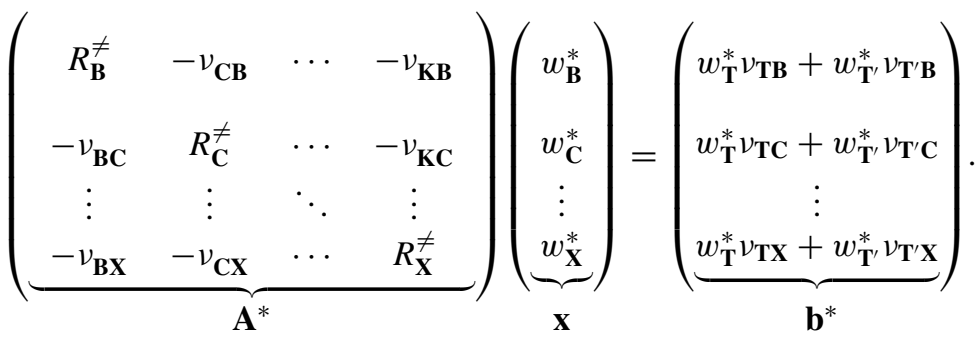

Using Cramer's rule, the solution to Eq. (31) is

$$
w_{\mathbf{P}}^{*}=\frac{B_{\mathbf{P}}^{*}}{B^{*}},
$$

where

$$
\begin{gathered}
B^{*}=\operatorname{det}\left(\mathbf{A}^{*}\right), \\
B_{\mathbf{P}}^{*}=\operatorname{det}\left(\mathbf{A}_{\mathbf{P}}^{*}\right)=\operatorname{det}\left(\mathbf{A}^{*}\left(\left[\mathbf{b}^{*}\right]_{c(\mathbf{P})}\right)\right) .
\end{gathered}
$$

Here, $\operatorname{det}\left(\mathbf{A}^{*}\right)$ is the determinant of $\mathbf{A}^{*}$ and $\mathbf{A}_{\mathbf{P}}^{*}=\mathbf{A}^{*}\left(\left[\mathbf{b}^{*}\right]_{c(\mathbf{P})}\right)$ is the matrix that results from substitution of column $c(\mathbf{P})$ in matrix $\mathbf{A}^{*}$ by vector $\mathbf{b}^{*}$, where $c(\mathbf{P})$ is the column corresponding to plane $\mathbf{P}$.

Since $\mathbf{b}^{*}$ in Eq. (31) may only contain linear combinations of the occupancies of the lower and upper terminal planes, $w_{\mathbf{T}}^{*}$ and $w_{\mathbf{T}^{\prime}}^{*}$, the determinants $B_{\mathbf{P}}^{*}$ can be expressed as linear combinations of those occupancies,

$$
B_{\mathbf{P}}^{*}=B_{\mathbf{P}, \mathbf{T} \square}^{*} w_{\mathbf{T}}^{*}+B_{\mathbf{P}, \square \mathbf{T}^{\prime}}^{*} w_{\mathbf{T}^{\prime}}^{*},
$$

where the coefficients $B_{\mathbf{P}, \mathbf{T} \square}^{*}$ and $B_{\mathbf{P}, \square \mathbf{T}^{\prime}}^{*}$ are defined as

$$
\begin{aligned}
& B_{\mathbf{P}, \mathbf{T} \square}^{*}=B_{\mathbf{P}}^{*}\left(w_{\mathbf{T}}^{*}=1, w_{\mathbf{T}^{\prime}}^{*}=0\right), \\
& B_{\mathbf{P}, \square \mathbf{T}^{\prime}}^{*}=B_{\mathbf{P}}^{*}\left(w_{\mathbf{T}}^{*}=0, w_{\mathbf{T}^{\prime}}^{*}=1\right) .
\end{aligned}
$$

Note that $B_{\mathbf{P}, \mathbf{T} \square}^{*}\left(B_{\mathbf{P}, \square \mathbf{T}^{\prime}}^{*}\right)$ is the value of the determinant $B_{\mathbf{P}}^{*}$ when the occupancy is 1 at the lower (upper) terminal plane and 0 at the upper (lower) terminal plane. See Sec. II E for examples of $B_{\mathbf{P}, \mathbf{T} \square}^{*}$ and $B_{\mathbf{P}, \square \mathbf{T}^{\prime}}^{*}$ in practice.

Now, inserting $B_{\mathbf{P}}^{*}$ from Eq. (35) into Eq. (32) shows that the occupancies of the inner planes $(\mathbf{P} \in \mathbf{K}=\{\mathbf{B}, \mathbf{C}, \ldots, \mathbf{G}\})$ are also linear combinations of the occupancies of the lower and upper boundary planes:

$$
w_{\mathbf{P}}^{*}=\frac{B_{\mathbf{P}, \mathbf{T} \square}^{*}}{B^{*}} w_{\mathbf{T}}^{*}+\frac{B_{\mathbf{P}, \square \mathbf{T}^{\prime}}^{*}}{B^{*}} w_{\mathbf{T}^{\prime}}^{*}
$$

Finally, substitution of Eq. (37) into Eq. (29) gives

$$
0=w_{\mathbf{T}}^{*} \Sigma_{\mathbf{P} \in \mathbf{F}}\left(v_{\mathbf{T P}}-\frac{B_{\mathbf{P}, \mathbf{T} \square}^{*}}{B^{*}} v_{\mathbf{P T}}\right)-w_{\mathbf{T}^{\prime}}^{*} \Sigma_{\mathbf{P} \in \mathbf{F}} \frac{B_{\mathbf{P}, \square \mathbf{T}^{\prime}}^{*}}{B^{*}} v_{\mathbf{P T}} .
$$

Equation (38) is instrumental. Comparing it to Eq. (30) directly provides the searched expressions for the forward and backward effective hop rates:

$$
\begin{aligned}
v_{\mathbf{T}, l}^{*} & =v_{\mathbf{T T}^{\prime}, l}^{+}=\sum_{\mathbf{P} \in \mathbf{F}} v_{\mathbf{T P}}-\sum_{\mathbf{P} \in \mathbf{F}} \frac{B_{\mathbf{P}, \mathbf{T} \square}^{*}}{B^{*}} v_{\mathbf{P T}} \\
& =v_{\mathbf{T}^{\prime} \mathbf{T}, l}^{-}=\sum_{\mathbf{P} \in \mathbf{F}} \frac{B_{\mathbf{P}, \square \mathbf{T}^{\prime}}^{*}}{B^{*}} \nu_{\mathbf{P T}} .
\end{aligned}
$$

The first term in Eq. (39) $\left(\sum_{\mathbf{P} \in \mathbf{F}} \nu_{\mathbf{T P}}\right)$ contains the rates of all direct hops from the lower terminal plane $\mathbf{T}$ to all destination planes $(\mathbf{P} \in \mathbf{F})$ across the special plane $\mathbf{S}$. The second term $\left(\sum_{\mathbf{P} \in \mathbf{F}}\left(B_{\mathbf{P}, \mathbf{T}}^{*} / B^{*}\right) \nu_{\mathbf{P T}}\right)$ contains the rates for all the hops from those destination planes $(\mathbf{P} \in \mathbf{F})$ to the lower terminal plane $\mathbf{T}$ across $\mathbf{S}$. Every rate $\left(\nu_{\mathbf{P T}}\right)$ is multiplied by the coefficient $B_{\mathbf{P}, \mathbf{T} \square}^{*} / B^{*}$. The second term is negative because the hops occur in the opposite direction to those in the first term. In Eq. (40) the first term is not present and the second term uses the opposite sign. The second term in Eq. (39) differs from the one in Eq. (40) in the use of different coefficients, namely, $\left(B_{\mathbf{P}, \mathbf{T} \square}^{*} / B^{*}\right)$ in Eq. (39) and $\left(B_{\mathbf{P}, \square \mathbf{T}^{\prime}}^{*} / B^{*}\right)$ in Eq. (40).

Equations (39) and (40) provide two equivalent expressions to determine the effective hop rate $v_{\mathbf{T T}, l}^{*}$ directly from the hop rates across plane $\mathbf{S}$ and the determinants $B^{*}=\operatorname{det}\left(\mathbf{A}^{*}\right)$ and $B_{\mathbf{P}}^{*}=\operatorname{det}\left(\mathbf{A}_{\mathbf{p}}^{*}\right)$, evaluated at the lower terminal plane $\mathbf{T}$ to obtain $B_{\mathbf{P}, \mathbf{T} \square}^{*}$ [Eq. (39)] or at the upper terminal plane $\mathbf{T}^{\prime}$ to obtain $B_{\mathbf{P}, \square \mathbf{T}^{\prime}}^{*}$ [Eq. (40)].

Alternatively, if we define $\mathbf{M}$ as the inverse of $\mathbf{A}^{*}$, the coefficients can be calculated using

$$
\frac{B_{\mathbf{P}, \mathbf{T} \square}^{*}}{B^{*}}=\sum_{\mathbf{Q} \in \mathbf{K}} M_{\mathbf{P Q}} \nu_{\mathbf{T Q}}, \quad \frac{B_{\mathbf{P}, \square \mathbf{T}^{\prime}}^{*}}{B^{*}}=\sum_{\mathbf{Q} \in \mathbf{K}} M_{\mathbf{P Q}}{ }^{\nu_{\mathbf{T}^{\prime}} \mathbf{Q}}
$$

In summary, we have shown that the effective hop rate between the terminals of a unit cell can be determined by (i) considering the net flux of particles across the special plane S, (ii) expressing the occupancies of the destination planes in terms of the occupancies of the lower and upper terminals, (iii) substituting the resulting expressions into the net flux, thus leading to an expression that contains only the occupancies of the lower and upper terminals, and (iv) reading off the effective hop rates as the coefficients that multiply those occupancies.

\section{E. Example: Propagation along $X$}

In this section we derive Eqs. (39) and (40) for propagation along $X$ in the unit cell shown in Fig. 2(f). First, using Eq. (3) with $j=1$ we determine all the site occupancies,

$$
\left.\begin{array}{ll}
w_{1}=\frac{v_{21} v_{31} v_{41}}{B}, & w_{2}=\frac{2 v_{12} v_{31} v_{41}}{B}, \\
w_{3}=\frac{\nu_{21} v_{13} v_{41}}{B}, & w_{4}=\frac{2 \nu_{21} v_{31} v_{14}}{B},
\end{array}\right\}
$$


with $B=v_{21} v_{31} v_{41}+2 v_{12} v_{31} v_{41}+v_{21} v_{13} v_{41}+2 v_{21} v_{31} v_{14}$. Thus, the effective site occupancies are

$$
\left.\begin{array}{l}
w_{1}^{*}=w_{1^{\prime}}^{*}=\frac{w_{1}}{1}=\frac{v_{21} v_{31} v_{41}}{B}, \\
w_{2}^{*}=w_{2^{\prime}}^{*}=\frac{w_{2}}{2}=\frac{v_{12} v_{31} v_{41}}{B}, \\
w_{3}^{*}=w_{3^{\prime}}^{*}=\frac{w_{3}}{1}=\frac{\nu_{21} v_{13} v_{41}}{B}, \\
w_{4}^{*}=w_{4^{\prime}}^{*}=\frac{w_{4}}{2}=\frac{\nu_{21} \nu_{31} v_{14}}{B} .
\end{array}\right\}
$$

Correspondingly, the occupancies of the terminal plane $\left(\mathbf{T}=\mathbf{A}\right.$ or $\left.\mathbf{T}^{\prime}=\mathbf{A}^{\prime}\right)$ and inner planes $(\mathbf{B}$ and $\mathbf{C})$ are

$$
\left.\begin{array}{l}
w_{\mathbf{A}}^{*}=w_{\mathbf{A}^{\prime}}^{*}=w_{1}^{*}+w_{3}^{*}=\frac{v_{21} v_{31} v_{41}+v_{21} v_{13} v_{41}}{B}, \\
w_{\mathbf{B}}^{*}=w_{\mathbf{C}}^{*}=w_{2}^{*}+w_{4}^{*}=\frac{v_{12} v_{31} v_{41}+v_{21} v_{31} v_{14}}{B} .
\end{array}\right\}
$$

Note that $w_{\mathbf{A}}^{*}+w_{\mathbf{B}}^{*}+w_{\mathbf{C}}^{*}=1$. Finally, the in-plane site occupancies are (using $v_{12} v_{24}^{v} v_{41}=v_{14} v_{42}^{v} v_{21}$; see Sec. PI-II D):

$$
\left.\begin{array}{l}
\hat{w}_{1}=\frac{w_{1}^{*}}{w_{\mathbf{A}}^{*}}=\frac{v_{31}}{v_{13}+v_{31}}, \\
\hat{w}_{2}=\frac{w_{2}^{*}}{w_{\mathbf{B}}^{*}}=\frac{v_{41} v_{12}}{v_{41} v_{12}+v_{21} v_{14}}=\frac{v_{42}^{v}}{v_{24}^{v}+v_{42}^{v}}, \\
\hat{w}_{3}=\frac{w_{3}^{*}}{w_{\mathbf{A}}^{*}}=\frac{v_{13}}{v_{13}+v_{31}}, \\
\hat{w}_{4}=\frac{w_{4}^{*}}{w_{\mathbf{B}}^{*}}=\frac{v_{21} v_{14}}{v_{41} v_{12}+v_{21} v_{14}}=\frac{v_{24}^{v}}{v_{24}^{v}+v_{42}^{v}} .
\end{array}\right\}
$$

Equation (45) shows that normally the in-plane occupancies can be expressed in terms of the hop rates along the chosen plane. Now, to simplify the presentation, let us define the following names for the rates between the planes:

$$
\left.\begin{array}{l}
p=v_{\mathbf{B A}}=v_{\mathbf{C A}^{\prime}}=\hat{w}_{2}\left(v_{21}+v_{23}\right)+\hat{w}_{4}\left(v_{41}+v_{43}\right), \\
q=v_{\mathbf{B C}}=v_{\mathbf{C B}}=\hat{w}_{2} v_{2}+\hat{w}_{4} v_{4}, \\
r=v_{\mathbf{A B}}=v_{\mathbf{A}^{\prime} \mathbf{C}}=\hat{w}_{1}\left(v_{12}+v_{14}\right)+\hat{w}_{3}\left(v_{32}+v_{34}\right),
\end{array}\right\}
$$

where $v_{2}=v_{22}^{h}+2 v_{22}^{d}+2 v_{24}^{d}$ and $v_{4}=v_{44}^{h}+2 v_{44}^{d}+2 v_{42}^{d}$. Then, due to detailed balance, the net flux through plane $\mathbf{S}$ along $X$ in Fig. 2(f) must be 0 :

$$
0=J_{\mathbf{S}}^{x+}-J_{\mathbf{S}}^{x-}=w_{\mathbf{A}}^{*} v_{\mathbf{A B}}-w_{\mathbf{B}}^{*} v_{\mathbf{B A}} .
$$

In terms of the forward and backward effective hop rates, $\nu_{\mathbf{A A}^{\prime}, x}^{+}$ and $v_{\mathbf{A}^{\prime} \mathbf{A}, x}^{-}$, the net flux can also be written as

$$
0=w_{\mathbf{A}}^{*} v_{\mathbf{A A}^{\prime}, x}^{+}-w_{\mathbf{A}^{\prime}}^{*} v_{\mathbf{A}^{\prime} \mathbf{A}, x}^{-} .
$$

Based on Eqs. (47) and (48), we would like to express $w_{\mathbf{B}}^{*}$ in terms of $w_{\mathbf{A}}^{*}$ and $w_{\mathbf{A}^{\prime}}^{*}$. To do this, we write down Eq. (31) (detailed balance) for all inner planes $\mathbf{P} \in\{\mathbf{B}, \mathbf{C}\}$. In matrix form, this reads

$$
(\underbrace{\left.\begin{array}{cc}
\mathrm{p}+q & -q \\
-q & \mathrm{p}+q
\end{array}\right)}_{\mathbf{A}^{*}}(\underbrace{w_{\mathbf{B}}^{*}}_{\mathbf{X}} \begin{array}{l}
\underbrace{*}_{\mathbf{C}}
\end{array})=\left(\begin{array}{c}
w_{\mathbf{A}}^{*} r \\
\underbrace{*}_{\mathbf{A}^{\prime}} r
\end{array}\right) .
$$

Since $\mathbf{b}^{*}$ in Eq. (49) [cf. Eq. (31)] contains only linear terms in the occupancies of the lower and upper terminal planes, $w_{\mathbf{A}}^{*}$ and $w_{\mathbf{A}^{\prime}}^{*}$, the occupancies of the inner planes $(\mathbf{P} \in \mathbf{K}=\{\mathbf{B}, \mathbf{C}\})$ are also linear combinations of the occupancies of the lower and upper boundary planes,

$$
w_{\mathbf{P}}^{*}=\frac{B_{\mathbf{P}, \mathbf{A} \square}^{*}}{B^{*}} w_{\mathbf{A}}^{*}+\frac{B_{\mathbf{P}, \square \mathbf{A}^{\prime}}^{*}}{B^{*}} w_{\mathbf{A}^{\prime}}^{*}
$$

where all symbols are defined in Eqs. (33)-(36). Here

$$
\begin{gathered}
B^{*}=\operatorname{det}\left(\mathbf{A}^{*}\right)=\left|\begin{array}{cc}
p+q & -q \\
-q & p+q
\end{array}\right|=p(p+2 q), \\
B_{\mathbf{B}}^{*}=\operatorname{det}\left(\mathbf{A}^{*}\left(\left[\mathbf{b}^{*}\right]_{1}\right)\right)=\left|\begin{array}{cc}
w_{\mathbf{A}}^{*} r & -q \\
w_{\mathbf{A}^{\prime}}^{*} r & \mathrm{p}+q
\end{array}\right| \\
=r(p+q) w_{\mathbf{A}}^{*}+r q w_{\mathbf{A}^{\prime}}^{*}, \\
B_{\mathbf{C}}^{*}=\operatorname{det}\left(\mathbf{A}^{*}\left(\left[\mathbf{b}^{*}\right]_{2}\right)\right)=\left|\begin{array}{cc}
\mathrm{p}+q & w_{\mathbf{A}}^{*} r \\
-q & w_{\mathbf{A}^{\prime}}^{*} r
\end{array}\right| \\
=r q w_{\mathbf{A}}^{*}+r(p+q) w_{\mathbf{A}^{\prime}}^{*}, \\
B_{\mathbf{B}, \mathbf{A} \square}^{*}=B_{\mathbf{B}}^{*}\left(w_{\mathbf{A}}^{*}=1, w_{\mathbf{A}^{\prime}}^{*}=0\right)=r(p+q), \\
B_{\mathbf{B}, \square \mathbf{A}^{\prime}}^{*}=B_{\mathbf{P}^{*}}^{*}\left(w_{\mathbf{A}}^{*}=0, w_{\mathbf{A}^{\prime}}^{*}=1\right)=r p, \\
B_{\mathbf{C}, \mathbf{A} \square}^{*}=B_{\mathbf{C}}^{*}\left(w_{\mathbf{A}}^{*}=1, w_{\mathbf{A}^{\prime}}^{*}=0\right)=r q, \\
B_{\mathbf{C}, \square \mathbf{A}^{\prime}}^{*}=B_{\mathbf{P}}^{*}\left(w_{\mathbf{A}}^{*}=0, w_{\mathbf{A}^{\prime}}^{*}=1\right)=r(p+q) .
\end{gathered}
$$

Finally, substitution of Eq. (50) for plane $\mathbf{P}=\mathbf{B}$ into Eq. (47) gives

$$
0=w_{\mathbf{A}}^{*}\left(v_{\mathbf{A B}}-\frac{B_{\mathbf{B}, \mathbf{A} \square}^{*}}{B^{*}} v_{\mathbf{B} \mathbf{A}}\right)-w_{\mathbf{A}^{\prime}}^{*}\left(\frac{B_{\mathbf{B}, \square \mathbf{A}^{\prime}}^{*}}{B^{*}} v_{\mathbf{B A}}\right) .
$$

Comparing Eqs. (58) and (48) directly provides the expressions for the forward and backward effective hop rates:

$$
\begin{aligned}
v_{\mathbf{A} \mathbf{A}, x}^{*} & =v_{\mathbf{A A}^{\prime}, x}^{+}=v_{\mathbf{A B}}-\frac{B_{\mathbf{B}, \mathbf{A} \square}^{*}}{B^{*}} v_{\mathbf{B A}} \\
& =v_{\mathbf{A}^{\prime} \mathbf{A}, x}^{-}=\frac{B_{\mathbf{B}, \square \mathbf{A}^{\prime}}^{*}}{B^{*}} v_{\mathbf{B A} .}
\end{aligned}
$$

Equations (59) and (60) are examples of Eqs. (39) and (40) in practice. Note that $v_{\mathbf{A A}^{\prime}, x}^{+}$and $\nu_{\mathbf{A}^{\prime} \mathbf{A}, x}^{-}$should be equal, thus providing the same value for $v_{\mathbf{A A}, x}^{*}$. Substitution of the expressions for $B^{*}, B_{\mathbf{B}, \mathbf{A} \square}^{*}$, and $B_{\mathbf{B}, \square \mathbf{A}^{\prime}}^{*}$ from Eqs. (51), (54), and (55), and use of Eq. (46) finally gives

$$
v_{\mathbf{A A}, x}^{*}=\frac{r q}{p+2 q}=\frac{\left[\hat{w}_{1}\left(v_{12}+v_{14}\right)+\hat{w}_{3}\left(v_{32}+v_{34}\right)\right]\left[\hat{w}_{2} v_{2}+\hat{w}_{4} v_{4}\right]}{\hat{w}_{2}\left(v_{21}+v_{23}+2 v_{2}\right)+\hat{w}_{4}\left(v_{41}+v_{43}+2 v_{4}\right)} .
$$

Thus, using Eq. (28), the $X$ component of the diffusivity is (no cell-commensurate sites, $\mathbf{T}=\mathbf{A}$, and no crossers),

$$
D_{T, x}^{\theta \approx 0}=w_{\mathbf{A}}^{*} v_{\mathbf{A} \mathbf{A}, x}^{*}(2 a)^{2}=\frac{4 v_{21} v_{41}\left[v_{31}\left(v_{12}+v_{14}\right)+v_{13}\left(v_{32}+v_{34}\right)\right]\left[v_{42}^{d} v_{2}+v_{24}^{d} v_{4}\right]}{B\left[v_{42}^{d}\left(v_{21}+v_{23}+2 v_{2}\right)+v_{24}^{d}\left(v_{41}+v_{43}+2 v_{4}\right)\right]} a^{2},
$$


where we have used Eq. (45) to write out the values for the inplane occupancies ( $\hat{w}_{1}$ through $\left.\hat{w}_{4}\right), B$ is given in Eq. (42), and $v_{2}$ and $v_{4}$ are given in Eq. (46). Appendix 1 in the Supplemental Material [14] shows that the final formula for the diffusivity is independent of the choice of unit cell.

\section{F. Example: Propagation along $\boldsymbol{Y}$}

In this section we use Eqs. (39) and (40) to determine the effective hop rates for propagation along the $Y$ direction for the unit cell shown in Fig. 2(k). Since boundary crossings occur the unit cell has been decomposed into the primary and complementary cells, as shown in Figs. 2(l) and 2(m), respectively. Figure 2(c) shows that the hops $v_{11}, v_{33}, v_{22}^{v}$, $v_{22}^{d}, v_{44}^{v}$, and $v_{44}^{d}$ are cell-commensurate along $Y$, and, thus, their contribution to the diffusivity is well defined in Eq. (28), i.e., $\left(w_{1} v_{11}+w_{2} v_{22}^{v d}+w_{3} v_{33}+w_{4} v_{44}^{v d}\right) a^{2}$, where $v_{22}^{v d}=v_{22}^{v}+$ $v_{22}^{d}$ and $v_{44}^{v d}=v_{44}^{v}+v_{44}^{d}$. Below, we first determine $v_{\mathbf{T T}, y}^{*}$ for the primary cell and then $\nu_{\mathbf{X X}, y}^{*}$ for the complementary cell. Finally, we gather together all the contributions of Eq. (28) and provide an expression for the diffusivity, demonstrating that the expression is independent of the choice of primary and/or complementary unit cell.
The site occupancies and effective occupancies for Fig. 2(1) are given in Eqs. (42) and (43). The occupancies of the terminal plane $\left(\mathbf{T}=\mathbf{A}\right.$ or $\left.\mathbf{T}^{\prime}=\mathbf{A}^{\prime}\right)$ and inner planes $(\mathbf{B}, \mathbf{C}$, and $\mathbf{D})$ are

$$
\begin{aligned}
& w_{\mathbf{A}}^{*}=w_{\mathbf{A}^{\prime}}^{*}=w_{2 a}^{*}+w_{2 b}^{*}=w_{2}=\frac{2 v_{12} v_{31} v_{41}}{B}, \\
& w_{\mathbf{B}}^{*}=w_{3}^{*}=w_{3}=\frac{v_{21} v_{13} v_{41}}{B}, \\
& w_{\mathbf{C}}^{*}=w_{4 a}^{*}+w_{4 b}^{*}=w_{4}=\frac{2 v_{21} v_{31} v_{14}}{B}, \\
& w_{\mathbf{D}}^{*}=w_{1}^{*}=w_{1}=\frac{v_{21} v_{31} v_{41}}{B}
\end{aligned}
$$

Note that $w_{\mathbf{A}}^{*}+w_{\mathbf{B}}^{*}+w_{\mathbf{C}}^{*}+w_{\mathbf{D}}^{*}=1$. Similarly, the in-plane site occupancies are

$$
\begin{aligned}
& \hat{w}_{2 a}=\frac{w_{2 a}^{*}}{w_{\mathbf{A}}^{*}}=\frac{1}{2}, \quad \hat{w}_{2 b}=\frac{w_{2 b}^{*}}{w_{\mathbf{A}}^{*}}=\frac{1}{2}, \quad \hat{w}_{3}=\frac{w_{3}^{*}}{w_{\mathbf{B}}^{*}}=1, \\
& \left.\hat{w}_{4 a}=\frac{w_{4 a}^{*}}{w_{\mathbf{C}}^{*}}=\frac{1}{2}, \quad \hat{w}_{4 b}=\frac{w_{4 b}^{*}}{w_{\mathbf{C}}^{*}}=\frac{1}{2}, \quad \hat{w}_{1}=\frac{w_{1}^{*}}{w_{\mathbf{D}}^{*}}=1 .\right\}
\end{aligned}
$$

Now, using the in-plane occupancies [Eq. (64)], all the rates between the planes are as follows:

$$
\begin{array}{ll}
\text { Forward } & \text { Backward } \\
\hline v_{\mathbf{A B}}=\hat{w}_{2 a} v_{23}+\hat{w}_{2 b} v_{23}=v_{23} & \nu_{\mathbf{A}^{\prime} \mathbf{D}}=\hat{w}_{2 a^{\prime}} v_{21}+\hat{w}_{2 b^{\prime}} v_{21}=v_{21} \\
\nu_{\mathbf{B C}}=\hat{w}_{3} 2 v_{34}=2 v_{34} & \nu_{\mathbf{D C}}=\hat{w}_{1} 2 v_{14}=2 v_{14} \\
v_{\mathbf{C D}}=\hat{w}_{4 a} v_{41}+\hat{w}_{4 b} v_{41}=v_{41} & \nu_{\mathbf{C B}}=\hat{w}_{4 a} v_{43}+\hat{w}_{4 b} v_{43}=v_{43} \\
\nu_{\mathbf{D A}^{\prime}}=\hat{w}_{1} 2 v_{12}=2 v_{12} & \nu_{\mathbf{B A}}=\hat{w}_{3} 2 v_{32}=2 v_{32} \\
v_{\mathbf{A C}}=\hat{w}_{2 a} v_{24}^{v d}+\hat{w}_{2 b} v_{24}^{v d}=v_{24}^{v d} & v_{\mathbf{A}^{\prime} \mathbf{C}}=\hat{w}_{2 a^{\prime}} v_{24}^{v d}+\hat{w}_{2 b^{\prime}} v_{24}^{v d}=v_{24}^{v d} \\
v_{\mathbf{C A}}=\hat{w}_{4 a} v_{42}^{v d}+\hat{w}_{4 b} v_{42}^{v d}=v_{42}^{v d} & \nu_{\mathbf{C A}}=\hat{w}_{4 a} v_{42}^{v d}+\hat{w}_{4 b} v_{42}^{v d}=v_{42}^{v d}
\end{array}
$$

where $v_{24}^{v d}=v_{24}^{v}+v_{24}^{d}$ and $v_{42}^{v d}=v_{42}^{v}+v_{42}^{d}$. Then, using Eqs. (39) and (40), the effective hop rate between the terminal planes is given by

$$
\begin{aligned}
v_{\mathbf{A} \mathbf{A}, y}^{*} & =v_{\mathbf{A} \mathbf{A}^{\prime}, y}^{+}=\frac{v_{\mathbf{A} \mathbf{B}}}{2}+v_{\mathbf{A C}}-\frac{B_{\mathbf{B}, \mathbf{A} \square}^{*}}{B^{*}} \frac{\nu_{\mathbf{B A}}}{2}-\frac{B_{\mathbf{C}, \mathbf{A} \square}^{*} v_{\mathbf{C A}},}{B^{*},} \\
& =v_{\mathbf{A}^{\prime} \mathbf{A}, y}^{-}=\frac{B_{\mathbf{B}, \square \mathbf{A}^{\prime}}^{*}}{B^{*}} \frac{\nu_{\mathbf{B A}}}{2}+\frac{B_{\mathbf{C}, \square \mathbf{A}^{\prime}}^{*}}{B^{*}} v_{\mathbf{C A}},
\end{aligned}
$$

where the determinants $B^{*}, B_{\mathbf{B}, \mathbf{A} \square}^{*}, B_{\mathbf{C}, \mathbf{A} \square}^{*}, B_{\mathbf{B}, \square \mathbf{A}^{\prime}}^{*}$, and $B_{\mathbf{C}, \square \mathbf{A}^{\prime}}^{*}$ refer to the following matrix equation [detailed balance for the inner planes B, C, and D; Eq. (31)]:

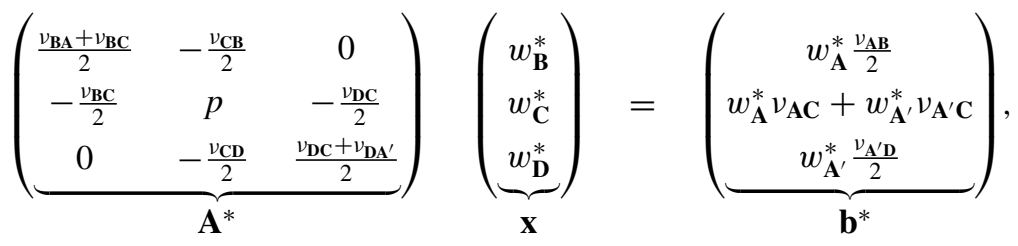

where $p=\left(v_{\mathbf{C B}}+v_{\mathbf{C D}}\right) / 2+v_{\mathbf{C A}}+v_{\mathbf{C A}^{\prime}}$. Note that in Eqs. (66)-(68) we have divided by 2 the hop rates indicated in Fig. 2(1), due to distributing the hops between the primary and the complementary cells (see Sec. II B 10). Using Eq. (65) to calculate the determinants and substituting them into Eqs. (66) and (67) finally gives (T= A)

$$
v_{\mathbf{T T}, y}^{*}=v_{\mathbf{A A}, y}^{*}=\frac{1}{2} \frac{\left[2 v_{24}^{v d}\left(v_{32}+v_{34}\right)+v_{23} v_{34}\right]\left[2 v_{42}^{v d}\left(v_{12}+v_{14}\right)+v_{41} v_{12}\right]}{\left[2 v_{42}^{v d}\left(v_{32}+v_{34}\right)+v_{43} v_{32}\right]\left(v_{12}+v_{14}\right)+\left[2 v_{42}^{v d}\left(v_{12}+v_{14}\right)+v_{41} v_{12}\right]\left(v_{32}+v_{34}\right)} \text {. }
$$


We focus now on obtaining the effective hop rate for the complementary cell shown in Fig. 2(m). The occupancies of the terminal plane $\left(\mathbf{T}=\mathbf{D}\right.$ or $\left.\mathbf{T}^{\prime}=\mathbf{D}^{\prime}\right)$ and inner planes $(\mathbf{A}, \mathbf{B}$, and $\mathbf{C})$ are

$$
\begin{aligned}
& w_{\mathbf{D}}^{*}=w_{\mathbf{D}^{\prime}}^{*}=w_{1}^{*}=w_{1}=\frac{\nu_{21} \nu_{31} v_{41}}{B}, \\
& w_{\mathbf{A}}^{*}=w_{2 a}^{*}+w_{2 b}^{*}=w_{2}=\frac{2 v_{12} v_{31} v_{41}}{B}, \\
& w_{\mathbf{B}}^{*}=w_{3}^{*}=w_{3}=\frac{v_{21} v_{13} v_{41}}{B}, \\
& w_{\mathbf{C}}^{*}=w_{4 a}^{*}+w_{4 b}^{*}=w_{4}=\frac{2 v_{21} v_{31} v_{14}}{B} .
\end{aligned}
$$

\begin{tabular}{|c|c|}
\hline Forward & Backward \\
\hline$v_{\mathbf{D A}}=\hat{w}_{1} 2 v_{12}=2 v_{12}$ & $v_{\mathbf{D}^{\prime} \mathbf{C}}=\hat{w}_{1} 2 v_{14}=2 v_{14}$ \\
\hline$v_{\mathbf{A B}}=\hat{w}_{2 a} v_{23}+\hat{w}_{2 b} \nu_{23}=v_{23}$ & $v_{\mathbf{C B}}=\hat{w}_{4 a} v_{43}+\hat{w}_{4 b} v_{43}=v_{43}$ \\
\hline$v_{\mathbf{B C}}=\hat{w}_{3} 2 v_{34}=2 v_{34}$ & $v_{\mathbf{B A}}=\hat{w}_{3} 2 v_{32}=2 v_{32}$ \\
\hline$v_{\mathbf{C D}^{\prime}}=\hat{w}_{4 a} v_{41}+\hat{w}_{4 b} v_{41}=v_{41}$ & $v_{\mathbf{A D}}=\hat{w}_{2 a^{\prime}} \nu_{21}+\hat{w}_{2 b^{\prime}} \nu_{21}=v_{21}$ \\
\hline$v_{\mathbf{D B}}=\hat{w}_{1} v_{13}=v_{13}$ & $v_{\mathbf{D}^{\prime} \mathbf{B}}=\hat{w}_{1} v_{13}=v_{13}$ \\
\hline$v_{\mathbf{B D}^{\prime}}=\hat{w}_{3} v_{31}=v_{31}$ & $v_{\mathbf{B D}}=\hat{w}_{3} v_{31}=v_{31}$ \\
\hline
\end{tabular}

In turn, the rates between the planes are as follows:

where we have used Eq. (64) to substitute the in-plane occupancies of the sites. Using now Eqs. (39) and (40), the effective hop rate between the terminals is

$$
\begin{aligned}
& v_{\mathbf{D D}, y}^{*}=v_{\mathbf{D D}^{\prime}, y}^{+}=\frac{v_{\mathbf{D A}}}{2}+v_{\mathbf{D B}}-\frac{B_{\mathbf{A}, \mathbf{D} \square}^{*}}{B^{*}} \frac{v_{\mathbf{A D}}}{2}-\frac{B_{\mathbf{B}, \mathbf{D} \square}^{*}}{B^{*}} v_{\mathbf{B D}}, \\
& =v_{\mathbf{D}^{\prime} \mathbf{D}, y}^{-}=\frac{B_{\mathbf{A}, \square \mathbf{D}^{\prime}}^{*}}{B^{*}} \frac{v_{\mathbf{A D}}}{2}+\frac{B_{\mathbf{B}, \square \mathbf{D}^{\prime}}^{*}}{B^{*}} v_{\mathbf{B D}},
\end{aligned}
$$

where the determinants $B^{*}, B_{\mathbf{A}, \mathbf{D} \square}^{*}, B_{\mathbf{B}, \mathbf{D} \square}^{*}, B_{\mathbf{A}, \square \mathbf{D}^{\prime}}^{*}$, and $B_{\mathbf{B}, \square \mathbf{D}^{\prime}}^{*}$ now refer to the following matrix equation for detailed balance at the inner planes $\mathbf{A}, \mathbf{B}$ and $\mathbf{C}$ :

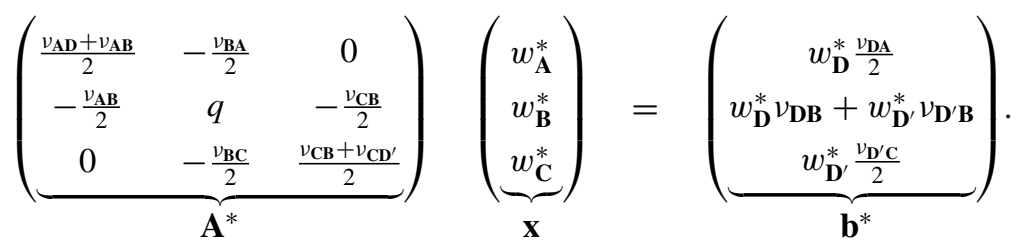

Here, $q=\left(v_{\mathbf{B A}}+v_{\mathbf{B C}}\right) / 2+v_{\mathbf{B D}}+v_{\mathbf{B D}^{\prime}}$. Making use of Eq. (71) to calculate the determinants and substituting them into Eqs. (72) and (73) finally leads to $(\mathbf{X}=\mathbf{D})$

$$
v_{\mathbf{X X}, y}^{*}=v_{\mathbf{D D}, y}^{*}=\frac{\left[v_{13}\left(v_{21}+v_{23}\right)+v_{12} v_{23}\right]\left[v_{31}\left(v_{41}+v_{43}\right)+v_{34} v_{41}\right]}{\left[v_{31}\left(v_{21}+v_{23}\right)+v_{32} v_{21}\right]\left(v_{41}+v_{43}\right)+\left[v_{31}\left(v_{41}+v_{43}\right)+v_{34} v_{41}\right]\left(v_{21}+v_{23}\right)} .
$$

Now, using Eq. (28), the $Y$ component of the diffusivity is

$$
D_{T, y}^{\theta \approx 0}=\left(w_{1} v_{11}+w_{2} v_{22}^{v d}+w_{3} v_{33}+w_{4} v_{44}^{v d}+w_{\mathbf{A}}^{*} v_{\mathbf{A A}, y}^{*}+w_{\mathbf{D}}^{*} v_{\mathbf{D D}, y}^{*}\right) a^{2},
$$

where the site occupancies $w_{1}, \ldots, w_{4}$ are given in Eq. (42), the effective plane occupancies $w_{\mathbf{A}}^{*}$ and $w_{\mathbf{D}}^{*}$ are given in Eq. (63), the effective hop rates $v_{\mathbf{A A}, y}^{*}$ and $v_{\mathbf{D D}, y}^{*}$ are given in Eqs. (69) and (75), respectively, and $v_{22}^{v d}=v_{22}^{v}+v_{22}^{d}, v_{44}^{v d}=v_{44}^{v}+v_{44}^{d}$, $v_{24}^{v d}=v_{24}^{v}+v_{24}^{d}$ and $v_{42}^{v d}=v_{42}^{v}+v_{42}^{d}$.

Note that Eq. (76) was obtained by using the primary cell shown in Fig. 2(1) and the lower complementary cell shown in Fig. 2(m). If we now focus on the use of the upper complementary cell, then, after distribution of the hops, the primary cell will look just like the one in Fig. 2(1) and the upper complementary cell will be similar to that in Fig. 2(m), but with the terminals coinciding with plane B. Thus, the diffusivity will be

$$
D_{T, y}^{\theta \approx 0}=\left(w_{1} v_{11}+w_{2} v_{22}^{v d}+w_{3} v_{33}+w_{4} v_{44}^{v d}+w_{\mathbf{A}}^{*} v_{\mathbf{A A}, y}^{*}+w_{\mathbf{B}}^{*} v_{\mathbf{B B}, y}^{*}\right) a^{2},
$$

where the effective hop rate between the $\mathbf{B}$ planes is calculated by a procedure similar to that for $v_{\mathbf{A A}, y}^{*}$ and $v_{\mathbf{D D}, y}^{*}$ above, leading to

$$
v_{\mathbf{B B}, y}^{*}=\frac{\left[v_{31}\left(v_{41}+v_{43}\right)+v_{34} v_{41}\right]\left[v_{13}\left(v_{21}+v_{23}\right)+v_{12} v_{23}\right]}{\left[v_{13}\left(v_{21}+v_{23}\right)+v_{12} v_{23}\right]\left(v_{41}+v_{43}\right)+\left[v_{13}\left(v_{41}+v_{43}\right)+v_{14} v_{43}\right]\left(v_{21}+v_{23}\right)} .
$$


Making use of the typical equalities between the products of the hop rates for the fundamental paths $\left(\operatorname{such}\right.$ as $v_{32} v_{21} v_{13}=v_{31} v_{12} v_{23}$ and $v_{34} v_{41} v_{13}=v_{31} v_{14} v_{43}$; see Sec. PI-2 D), it can be easily shown that the terms $w_{\mathbf{D}}^{*} v_{\mathbf{D D}, y}^{*}$ [in Eq. (76)] and $w_{\mathbf{B}}^{*} v_{\mathbf{B B}, y}^{*}$ [in Eq. (77)] are identical. This demonstrates that the diffusivity does not depend on the choice of lower/upper complementary unit cell.

Moreover, if we choose the unit cell shown in Fig. 2(h), then the diffusivity can be formulated as

$$
D_{T, y}^{\theta \approx 0}=\left(w_{1} v_{11}+w_{2} v_{22}^{v d}+w_{3} v_{33}+w_{4} v_{44}^{v d}+w_{\mathbf{B}}^{*} \nu_{\mathbf{B B}, y}^{*}+w_{\mathbf{C}}^{*} \nu_{\mathbf{C C}, y}^{*}\right) a^{2},
$$

where the effective hop rate between the $\mathbf{C}$ planes is calculated as

$$
v_{\mathbf{C C}, y}^{*}=\frac{1}{2} \frac{\left[2 v_{24}^{v d}\left(v_{32}+v_{34}\right)+v_{23} v_{34}\right]\left[2 v_{42}^{v d}\left(v_{12}+v_{14}\right)+v_{41} v_{12}\right]}{\left[2 v_{24}^{v d}\left(v_{32}+v_{34}\right)+v_{23} v_{34}\right]\left(v_{12}+v_{14}\right)+\left[2 v_{24}^{v d}\left(v_{12}+v_{14}\right)+v_{21} v_{14}\right]\left(v_{32}+v_{34}\right)} .
$$

Again, making use of $v_{23} v_{34} v_{42}^{v d}=v_{24}^{v d} v_{43} v_{32}$ and $v_{21} v_{14} v_{42}^{v d}=$ $v_{24}^{v d} v_{41} v_{12}$, one can easily show that the terms $w_{\mathbf{A}}^{*} v_{\mathbf{A A}, y}^{*}$ [in Eq. (77)] and $w_{\mathbf{C}}^{*} v_{\mathbf{C C}, y}^{*}$ [in Eq. (79)] are identical. This demonstrates that the diffusivity does not depend on the choice of primary unit cell.

\section{G. Algorithm (M-1)}

The procedure presented in Secs. II B-IID to obtain the effective hop rates can be summarized as the algorithm reported in Table I. The procedure is referred to as M-1 to differentiate it from an alternative method described in Sec. IV. As far as we can tell, procedure M-1 can be used to find the effective hop rates for any periodic system. This can be

TABLE I. Procedure M-1 to determine the effective hop rates $v_{\mathbf{T T}, l}^{*}$ and $v_{\mathbf{X X}, l}^{*}$.

Determine $w_{i}$ [site occupancies, Eq. (3)].

Define an orthorhombic unit cell.

(1) For every dimension $l(X, Y$, and $Z)$ :

Relabel all equivalent sites. Determine $w_{i}^{*}$ [effective occupancies of the relabeled sites, Eq. (6)].

Define the planes $\mathbf{A}, \mathbf{B}, \mathbf{C}, \ldots, \mathbf{X}, \mathbf{A}^{\prime}$.

Determine $w_{\mathbf{P}}^{*}$ [effective occupancies of planes, Eq. (7)].

Determine $\hat{w}_{i}$ [in-plane occupancies, Eq. (8)].

Determine $v_{\mathbf{P Q}}$ [hop rates between planes, Eq. (9)].

Consider the 1D representation. If boundary crossings occur, find the lower complementary cell (with the lower terminal at $\mathbf{X}$ ). Distribute the hops between primary and complementary cells following rules R1-R3 (Sec. II B 10).

(2) For every unit cell (primary/complementary):

Use $\mathbf{T}\left(\mathbf{T}^{\prime}\right)$ to denote the lower (upper) terminal plane. Use $\mathbf{K}$ to designate the set of inner planes.

Define plane $\mathbf{S}$ (Sec. II D). Determine which hops cross $\mathbf{S}$. Determine the set of destination planes $\mathbf{F}$.

Build matrix $\mathbf{A}^{*}$ and vector $\mathbf{b}^{*}$ for all inner planes $\mathbf{P} \in \mathbf{K}$ [Eq. (31)].

Calculate the determinants $B^{*}$ and $B_{\mathbf{P}}^{*}$ for every inner plane $\mathbf{P} \in \mathbf{K}$.

For the lower terminal $\mathbf{T}$, evaluate $B_{\mathbf{P}, \mathbf{T} \square}^{*}=B_{\mathbf{P}}^{*}\left(w_{\mathbf{T}}^{*}=1, w_{\mathbf{T}^{\prime}}^{*}=0\right)$.

For the upper terminal $\mathbf{T}^{\prime}$, evaluate $B_{\mathbf{P}, \square \mathbf{T}^{\prime}}^{*}=B_{\mathbf{P}}^{*}\left(w_{\mathbf{T}}^{*}=0, w_{\mathbf{T}^{\prime}}^{*}=1\right)$.

Determine $v_{\mathbf{T T}, l}^{*}$ [effective hop rate, Eqs. (39) and (40)]. (For the complementary cell this directly gives $v_{\mathbf{X X}, l}^{*}$ )

(2) End.

(1) End. done analytically (with pen and paper), if the system is simple enough, or numerically/symbolically using a computer, for very complex systems. The availability of two different (but equivalent) formulas [Eqs. (39) and (40)] can be used as an advantage to internally check whether the obtained solutions are correct.

\section{LOW COVERAGE DIFFUSIVITY FOR 1D SYSTEMS WITH ASYMMETRIC HOPS}

We now apply procedure M-1, summarized in Table I, to derive the effective hop rate and diffusivity for 1D systems with asymmetric hops to the nearest neighbors and no boundary crossings. As shown in Sec. IV, the expressions obtained here will provide an alternative procedure for formulating the effective hop rates (and, thus, the diffusivity) for systems with asymmetric hops in more dimensions.

\section{A. Effective hop rate for the longest path}

Figures 4(a)-4(c) show several examples of 1D periodic systems with asymmetric hops and a varying number of

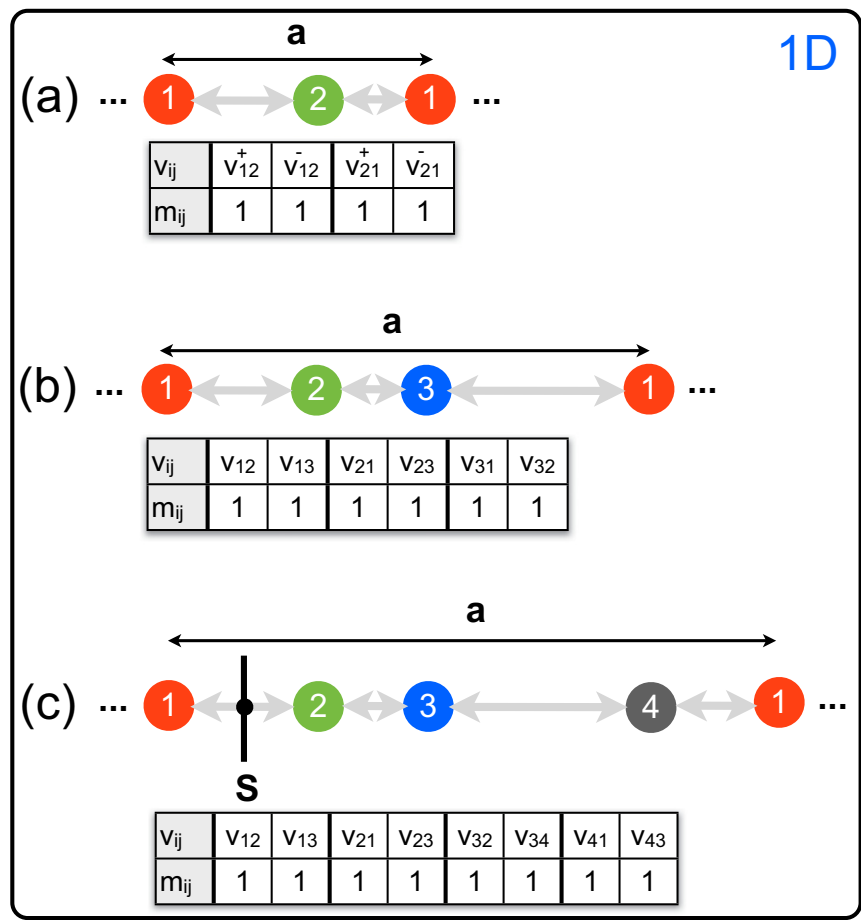

FIG. 4. One-dimensional systems with (a) $S=2$, (b) $S=3$, and (c) $S=4$ adsorption sites. Hops from any site are restricted to the forward and backward nearest neighbors. 
distinct sites: $S=2$ for Fig. 4(a), $S=3$ for Fig. 4(b), and $S=4$ for Fig. 4(c). Since all the sites within one unit cell are different, the effective occupancies for the sites are equal to their occupancies $\left(w_{i}^{*}=w_{i}\right)$. In addition, we may define one plane $\mathbf{P}$ per site $i$, thus having $w_{\mathbf{P}}^{*}=w_{i}^{*}=w_{i}$. As a result, we can treat every site as one plane and every plane as one site. Recalling from Sec. II B 9 that a $1 D$ path is simply a collection of consecutive hops and that the longest path is defined by having the length of the unit cell, the periodic systems in Fig. 4 can be considered as periodic paths, obtained by repeating the longest path for each system. Considering the system with four distinct planes [Fig. 4(c)] and placing the special plane $\mathbf{S}$ between plane 1 and plane 2, the effective hop rate is obtained by applying Eq. (39) [or (40)]:

$$
\begin{aligned}
v_{11, x}^{*} & =v_{11, x}^{+}=v_{12}-\frac{B_{2,1 \square}^{*}}{B^{*}} v_{21} \\
& =v_{11, x}^{-}=\frac{B_{2, \square 1^{\prime}}^{*}}{B^{*}} v_{21} .
\end{aligned}
$$

This means that we can focus on obtaining expressions for $B^{*}$ and $B_{2,1 \square}^{*}$ (or $B_{2, \square 1^{\prime}}^{*}$ ). For this purpose, we write Eq. (31) for all inner planes $\mathbf{K}=\{2,3,4\}$,

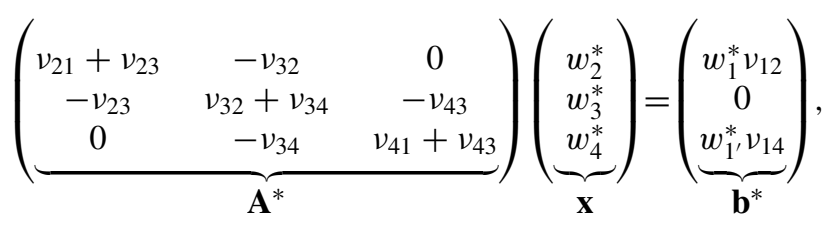

and substitute vector $\mathbf{b}^{*}$ (evaluated at $\left\{w_{1}^{*}, w_{1^{\prime}}^{*}\right\}=\{1,0\}$ ) as the first column in matrix $\mathbf{A}^{*}$ to get

$$
\mathbf{A}_{2,1 \square}^{*}=\left(\begin{array}{ccc}
v_{12} & -v_{32} & v_{12} \\
0 & v_{32}+v_{34} & -v_{43} \\
0 & -v_{34} & v_{41}+v_{43}
\end{array}\right) .
$$

Similarly, we substitute vector $\mathbf{b}^{*}(\{0,1\})$ as the first column in matrix $\mathbf{A}^{*}$ to get

$$
\mathbf{A}_{2, \square 1^{\prime}}^{*}=\left(\begin{array}{ccc}
0 & -v_{32} & v_{12} \\
0 & v_{32}+v_{34} & -v_{43} \\
v_{12} & -v_{34} & v_{41}+v_{43}
\end{array}\right)
$$

Thus, we obtain

$$
\begin{gathered}
B^{*}=\operatorname{det}\left(\mathbf{A}^{*}\right)=v_{23} v_{34} v_{41}+v_{43} v_{32} v_{21} \\
+v_{34} v_{41} v_{21}+v_{32} v_{21} v_{41}, \\
B_{2,1 \square}^{*}=\operatorname{det}\left(\mathbf{A}_{2,1 \square}^{*}\right)=v_{12}\left(v_{32} v_{41}+v_{32} v_{43}+v_{34} v_{41}\right), \\
B_{2, \square 1^{\prime}}^{*}=\operatorname{det}\left(\mathbf{A}_{2, \square 1^{\prime}}^{*}\right)=v_{14} v_{43} v_{32} .
\end{gathered}
$$

Replacing these into Eqs. (81) and (82) we obtain the forward and backward effective hop rates:

$$
v_{11, x}^{+}=\frac{v_{12} \nu_{23} \nu_{34} v_{41}}{v_{23} v_{34} v_{41}+v_{43} v_{32} v_{21}+v_{34} v_{41} v_{21}+v_{32} v_{21} v_{41}},
$$

$$
v_{11, x}^{-}=\frac{v_{14} \nu_{43} \nu_{32} \nu_{21}}{v_{23} \nu_{34} \nu_{41}+v_{43} \nu_{32} \nu_{21}+v_{34} \nu_{41} \nu_{21}+v_{32} \nu_{21} v_{41}} \text {. }
$$

These are equal $\left(v_{11, x}^{+}=v_{11, x}^{-}=v_{11, x}^{*}\right)$ due to detailed balance (zero net flux), thus requiring that $v_{12} v_{23} v_{34} v_{41}=v_{14} v_{43} v_{32} v_{21}$. This is satisfied for hop rates described using Boltzmann factors, as shown in Fig. PI-3(g).

For two and three distinct planes $[S=2$ and $S=3$; see Figs. 4(a) and 4(b)], applying the same procedure will lead to

$$
\begin{aligned}
v_{11, x}^{*} & =\frac{v_{12}^{+} v_{21}^{+}}{v_{21}^{+}+v_{21}^{-}}=\frac{v_{12}^{-} v_{21}^{-}}{v_{21}^{+}+v_{21}^{-}}, \\
v_{11, x}^{*} & =\frac{v_{12} v_{23} v_{31}}{v_{23} v_{31}+v_{32} v_{21}+v_{31} v_{21}} \\
& =\frac{v_{13} v_{32} v_{21}}{v_{23} v_{31}+v_{32} v_{21}+v_{31} v_{21}} .
\end{aligned}
$$

In general, for $S$ distinct planes we can write

$$
v_{i i, x}^{*}=\frac{\Pi_{p=1}^{S} v_{p q}}{\sum_{j=1}^{S} \Pi_{k=i+1}^{S+i-1} v_{l m}} \quad(i=1,2, \ldots, S),
$$

where the indices $l, m$, and $q$ are

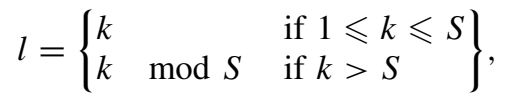

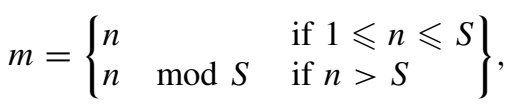

$$
\begin{aligned}
& n=\left\{\begin{array}{ll}
k-1 & \text { if } j+k<S+i+1 \\
k+1 & \text { if } j+k \geqslant S+i+1
\end{array}\right\}, \\
& q=\left\{\begin{array}{ll}
p+1 & \text { if } p<S \\
1 & \text { if } p=S
\end{array}\right\} .
\end{aligned}
$$

Equations (89)-(92) are identical to [and Eq. (93) is equivalent to] those for the composite rates reported by Condit [11] and Birnie [12]. Thus, for 1D systems, the effective hop rates defined in this study are reduced to the previously reported composite rates. Hence, the procedure summarized in Sec. II G for $\alpha \geqslant 1$ can be considered as a generalization of the 1D analysis reported by Condit [11]. Our analysis differs in the following aspects: (i) our expression for the positive/negative/net flux uses well-defined quantities to describe the probability that the random walker is at every site or plane. In fact, the occupancy $w_{i}$ for the site $i$ and the effective occupancy $w_{\mathbf{P}}^{*}$ for plane $\mathbf{P}$ describe the fraction of time that the random walker spends at that site or plane; (ii) our formalism provides explicit expressions for the occupancies [Eqs. (3), PI-(40), and PI-(41)] and effective occupancies [Eqs. (6)-(8)]; (iii) our expression for the net flux [Eq. (29)] is guaranteed to be 0 due to the general principle of detailed balance; (iv) we provide a general procedure/algorithm for determining the effective hop rates for any periodic system (procedure M-1, in Table I); and (v) our procedure applies to any number of dimensions and contains explicit expressions to determine the effective hop rates [Eqs. (39) and (40)].

\section{B. Effective hop rate for shorter paths}

Let us now consider a forward/backward path that is shorter than the longest path. An important result is that the effective hop rate for such a path is still provided by Eq. (93) (or the equivalent formulas for $S \leqslant 4$ ). As an example, using Eq. (91) the effective hop rate for the forward path 123 (backward path 
321) in Fig. 4(b) is

$$
v_{123}=v_{13}^{+}=\frac{v_{12} v_{23}}{v_{21}+v_{23}}\left(v_{321}=v_{31}^{-}=\frac{v_{32} v_{21}}{v_{23}+v_{21}}\right) .
$$

Another important result is that the longest path can be split at any plane and the total effective hop rate (for the longest path) can still be calculated by using Eq. (91) for the resulting left and right sections of the path. As an example, we can split the path 1231 in Fig. 4(b) at plane 3 and describe the effective hop rate using the left and right sections (123 and 31, respectively):

$$
\begin{aligned}
v_{11}^{+} & =\frac{v_{123} v_{31}}{v_{321}+v_{31}}=\frac{\frac{v_{12} v_{23}}{v_{21}+v_{23}} v_{31}}{\frac{v_{32} \nu_{21}}{v_{23}+v_{21}}+v_{31}} \\
& =\frac{v_{12} v_{23} v_{31}}{v_{32} v_{21}+v_{31} v_{21}+v_{31} v_{23}} .
\end{aligned}
$$

Equivalently, we can split the path at plane 2 and describe the effective hop rate using the left section (12) and the right section (231):

$$
\begin{aligned}
v_{11}^{+} & =\frac{v_{12} v_{231}}{v_{21}+v_{231}}=\frac{v_{12} \frac{v_{23} v_{31}}{v_{32}+v_{31}}}{v_{21}+\frac{v_{23} \nu_{31}}{v_{32}+v_{31}}} \\
& =\frac{v_{12} \nu_{23} v_{31}}{v_{32} v_{21}+v_{31} v_{21}+v_{31} v_{23}} .
\end{aligned}
$$

Note that Eqs. (96) and (97) are identical and equal to Eq. (92). Similarly, longer paths can be split in many ways, all giving the same effective hop rate. For instance, if we split the path 12341 in Fig. 4(c) at planes 3 and 4, the effective hop rate will be

$$
\begin{aligned}
v_{11}^{+} & =\frac{v_{123} v_{341}}{v_{321}+v_{341}}=\frac{\frac{v_{12} v_{23}}{v_{21}+v_{23}} \frac{v_{34} v_{41}}{v_{41}+v_{43}}}{\frac{v_{32} v_{21}+\frac{v_{34} v_{41}}{v_{21}+v_{23}}}{v_{41}+v_{43}}} \quad \text { (at 3) } \\
& =\frac{v_{1234} v_{41}}{v_{4321}+v_{41}}=\frac{\frac{v_{12} v_{23} v_{34}}{v_{23} v_{34}+v_{32} v_{21}+v_{21} v_{34}} v_{41}}{\frac{v_{43} v_{32} v_{21}}{v_{23} v_{34}+v_{32} v_{21}+v_{21} v_{34}}+v_{41}} \quad \text { (at 4) } \\
& =\frac{v_{12} v_{23} v_{34} v_{41}}{v_{23} v_{34} v_{41}+v_{43} v_{32} v_{21}+v_{34} v_{41} v_{21}+v_{32} v_{21} v_{41}} .
\end{aligned}
$$

Note that Eq. (100) is identical to Eq. (89). From these examples we conclude that, given a $1 \mathrm{D}$ periodic system and unit cell, the longest path can be split in multiple ways, all giving the same effective hop rate. Together with the additive formula for the hop rates of parallel paths between two planes [Eq. (17)], this result allows splitting long paths into short paths (sections) so that every section is hop commensurate to a long hop, as described below (Sec. IV).

\section{Diffusivity}

For completeness, we determine the diffusivity for the 1D systems in Fig. 4. Following with the example for $S=4$ [Fig. 4(c)], we first determine the occupancies using Eqs. PI-(40) and PI-(41):

$$
\begin{gathered}
w_{i}=\frac{B_{i}}{B_{1}+B_{2}+B_{3}+B_{4}} \quad(i=1,2, \ldots, 4), \\
B_{1}=\operatorname{det}\left(\mathbf{A}_{1}\right)=\left|\begin{array}{ccc}
v_{21}+v_{23} & -v_{32} & 0 \\
-v_{23} & v_{32}+v_{34} & -v_{43} \\
0 & -v_{34} & v_{41}+v_{43}
\end{array}\right| \\
=v_{23} v_{34} v_{41}+v_{43} v_{32} v_{21}+v_{34} v_{41} v_{21}+v_{32} v_{21} v_{41},
\end{gathered}
$$

$$
\begin{aligned}
B_{2} & =\operatorname{det}\left(\mathbf{A}_{2}\right)=\left|\begin{array}{ccc}
v_{12}+v_{14} & 0 & -v_{41} \\
0 & v_{32}+v_{34} & -v_{43} \\
-v_{14} & -v_{34} & v_{41}+v_{43}
\end{array}\right| \\
& =v_{34} v_{41} v_{12}+v_{14} v_{43} v_{32}+v_{41} v_{12} v_{32}+v_{43} v_{32} v_{12}, \\
B_{3} & =\operatorname{det}\left(\mathbf{A}_{3}\right)=\left|\begin{array}{ccc}
v_{12}+v_{14} & -v_{21} & -v_{41} \\
-v_{12} & v_{21}+v_{23} & 0 \\
-v_{14} & 0 & v_{41}+v_{43}
\end{array}\right| \\
& =v_{41} v_{12} v_{23}+v_{21} v_{14} v_{43}+v_{12} v_{23} v_{43}+v_{14} v_{43} v_{23}, \\
B_{4} & =\operatorname{det}\left(\mathbf{A}_{4}\right)=\left|\begin{array}{ccc}
v_{12}+v_{14} & -v_{21} & 0 \\
-v_{12} & v_{21}+v_{23} & -v_{32} \\
0 & -v_{23} & v_{32}+v_{34}
\end{array}\right| \\
& =v_{12} v_{23} v_{34}+v_{32} v_{21} v_{14}+v_{23} v_{34} v_{14}+v_{21} v_{14} v_{34} .
\end{aligned}
$$

Note that other expressions can be obtained for $w_{1}$ through $w_{4}$ by using Eq. (3). Nevertheless, all expressions are equivalent under typical fundamental path transformations (see Sec. PIIID). Now, noting that $B^{*}$ in Eq. (86) is equal to $B_{1}$ in Eq. (102), the diffusivity is $\left(w_{i}^{*}=w_{i}\right.$, since $n_{i}=1$ for all $\left.i\right)$

$$
\begin{aligned}
D_{T}^{\theta \approx 0} & =\left(\Sigma_{i \in \mathcal{T}} w_{i}^{*} v_{i i, x}^{*}\right) a^{2} \\
& =w_{1} v_{11, x}^{*} a^{2} \\
& =\frac{B_{1}}{B_{1}+B_{2}+B_{3}+B_{4}} \frac{v_{12} v_{23} v_{34} v_{41}}{B_{1}} a^{2} \\
& =\frac{v_{12} v_{23} v_{34} v_{41}}{B_{1}+B_{2}+B_{3}+B_{4}} a^{2} .
\end{aligned}
$$

Note that we can also use the following expressions:

$$
\begin{aligned}
D_{T}^{\theta \approx 0} & =w_{2} v_{22, x}^{*} a^{2} \\
& =\frac{B_{2}}{B_{1}+B_{2}+B_{3}+B_{4}} \frac{\nu_{23} v_{34} v_{41} v_{12}}{B_{2}} a^{2} \\
& =w_{3} v_{33, x}^{*} a^{2} \\
& =\frac{B_{3}}{B_{1}+B_{2}+B_{3}+B_{4}} \frac{v_{34} v_{41} v_{12} v_{23}}{B_{3}} a^{2} \\
& =w_{4} v_{44, x}^{*} a^{2} \\
& =\frac{B_{4}}{B_{1}+B_{2}+B_{3}+B_{4}} \frac{v_{41} v_{12} v_{23} v_{34}}{B_{4}} a^{2} .
\end{aligned}
$$

Similarly, for 1D systems with $S$ distinct sites, in general, the diffusivity is

$$
\begin{aligned}
D_{T}^{\theta \approx 0} & =w_{i} v_{i i, x}^{*} a^{2} \quad(i=1,2, \ldots, S) \\
& =\frac{B_{i}}{\sum_{i=1}^{S} B_{i}} \frac{\Pi_{p=1}^{S} v_{p q}}{B_{i}} a^{2} \\
& =\frac{\prod_{p=1}^{S} v_{p q}}{\sum_{i=1}^{S} B_{i}} a^{2} \\
& =\frac{\Pi_{p=1}^{S} v_{p q}}{\sum_{i=1}^{S}\left(\sum_{j=1}^{S} \Pi_{k=i+1}^{S+i-1} v_{l m}\right)} a^{2},
\end{aligned}
$$

where the indices $l, m$, and $q$ are given in Eq. (94) in terms of the indices $i, j, k$, and $p$. 


\section{DETERMINATION OF THE EFFECTIVE HOP RATES BASED ON 1D FORMULAS}

Regarding Eqs. (39) and (40), this section provides an alternative procedure to formulate the effective hop rate $\left(v_{\mathbf{T T}, l}^{*}\right.$ or $\left.v_{\mathbf{X X}, l}^{*}\right)$ - and thus obtain the diffusivity-by using combinations of the 1D formulas derived in Sec. III. The key idea is to describe $v_{\mathbf{T T}, l}^{*}$ and $v_{\mathbf{X X}, l}^{*}$ using Eq. (93) for $S$ different planes. If long hops with hop-commensurate paths occur, the hop rate of every long hop and that of its hop-commensurate path must be added together in order to correctly calculate the total hop rate between the planes separated by the long hop (see Sec. II B 9). Although this operation may split long paths into shorter paths, this does not affect the contribution to the diffusivity from the long paths (see Sec. IIIB). If boundary crossings occur, Eq. (93) is applied to the primary and complementary cells separately. If the number of distinct planes $S$ is 2, 3, or 4 we can directly use Eq. (91), (92), and (89), respectively.

\section{A. Example: Propagation along $X$ revisited}

Let us consider again the example shown in Fig. 2. Focusing first on the $1 \mathrm{D}$ representation for the propagation along $X$, as shown in Fig. 2(f), we conclude that there are three distinct planes (A, B, and $\mathbf{C}$ ). Thus, $S=3$ and we can write the effective hop rate using Eq. (92):

$$
\begin{aligned}
v_{\mathbf{A A}, x}^{*} & =\frac{v_{\mathbf{A B}} v_{\mathbf{B C}} v_{\mathbf{C A} \mathbf{A}^{\prime}}}{v_{\mathbf{B C}} v_{\mathbf{C A}}+v_{\mathbf{C B}} v_{\mathbf{B A}}+v_{\mathbf{B A}} v_{\mathbf{C A}}} \\
& =\frac{v_{\mathbf{A B}} v_{\mathbf{B B}} v_{\mathbf{B A}}}{v_{\mathbf{B B}} v_{\mathbf{B A}}+v_{\mathbf{B B}} v_{\mathbf{B A}}+v_{\mathbf{B A}} v_{\mathbf{B A}}}=\frac{v_{\mathbf{A B}} v_{\mathbf{B B}}}{v_{\mathbf{B A}}+2 v_{\mathbf{B B}}},
\end{aligned}
$$

where we have used that planes $\mathbf{B}$ and $\mathbf{C}$ ( $\mathbf{A}$ and $\mathbf{A}^{\prime}$ ) are equivalent. Using Eq. (9), the hop rates between the planes are

$$
\begin{gathered}
v_{\mathbf{A B}}=\hat{w}_{1}\left(v_{12}+v_{14}\right)+\hat{w}_{3}\left(v_{32}+v_{34}\right), \\
v_{\mathbf{B B}}=\hat{w}_{2} v_{2}+\hat{w}_{4} v_{4}, \\
v_{\mathbf{B A}}=\hat{w}_{2}\left(v_{21}+v_{23}\right)+\hat{w}_{4}\left(v_{41}+v_{43}\right),
\end{gathered}
$$

with $v_{2}=v_{22}^{h}+2 v_{22}^{d}+2 v_{24}^{d}$ and $v_{4}=v_{44}^{h}+2 v_{44}^{d}+2 v_{42}^{d}$. Substituting Eqs. (115)-(117) into Eq. (114), we obtain

$$
v_{\mathbf{A A}, x}^{*}=\frac{\left[\hat{w}_{1}\left(v_{12}+v_{14}\right)+\hat{w}_{1}\left(v_{32}+v_{34}\right)\right]\left[\hat{w}_{2} v_{2}+\hat{w}_{4} v_{4}\right]}{\hat{w}_{2}\left(v_{21}+v_{23}+2 v_{2}\right)+\hat{w}_{4}\left(v_{41}+v_{43}+2 v_{4}\right)},
$$

which is the same expression as derived in Sec. II E (Eq. 61). Thus, the diffusivity is also given by Eq. (62). In conclusion, the use of the 1D formula [Eq. (92)] provides a fast approach to obtaining the effective hop rate and, in turn, also the diffusivity.

\section{B. Example: Propagation along $Y$ revisited}

We focus now on the propagation along $Y$, using the $1 \mathrm{D}$ representation of the primary and complementary cells shown in Figs. 2(l) and 2(m). This system features hopcommensurate paths, and thus, the rate of every long hop and its commensurate path must be added together in order to correctly calculate the total hop rate between the planes separated by the long hop (see Sec. II B 9). For instance, considering the primary cell first [Fig. 2(1)], the hop rate from plane $\mathbf{A}$ to plane $\mathbf{C}$ is written

$$
\begin{aligned}
v_{\mathbf{A C}}^{+} & =v_{\mathbf{A C}}+\frac{1}{2} v_{\mathbf{A B C}} \\
& =v_{\mathbf{A C}}+\frac{1}{2} \frac{v_{\mathbf{A B}} v_{\mathbf{B C}}}{v_{\mathbf{B A}}+v_{\mathbf{B C}}} \\
& =\hat{w}_{2 a} v_{24}^{v d}+\hat{w}_{2 b} v_{24}^{v d}+\frac{1}{2} \frac{\left(\hat{w}_{2 a} v_{23}+\hat{w}_{2 b} v_{23}\right) \hat{w}_{3} 2 v_{34}}{\hat{w}_{3} 2 v_{32}+\hat{w}_{3} 2 v_{34}} \\
& =\frac{2 v_{24}^{v d}\left(v_{32}+v_{34}\right)+v_{23} v_{34}}{2\left(v_{32}+v_{34}\right)}
\end{aligned}
$$

where we have used Eq. (91) to describe the rate for path $\mathbf{A B C}$ : $v_{\mathbf{A B C}}=v_{\mathbf{A B}} v_{\mathbf{B C}} /\left(v_{\mathbf{B A}}+v_{\mathbf{B C}}\right)$. Similarly, based on Eq. (9), we have written $v_{\mathbf{A C}}=\hat{w}_{2 a} v_{24}^{v d}+\hat{w}_{2 b} v_{24}^{v d}=v_{24}^{v d}$ and $v_{\mathbf{A B}}=$ $\hat{w}_{2 a} v_{23}+\hat{w}_{2 b} v_{23}=v_{23}$, with $\hat{w}_{2 a}=\hat{w}_{2 b}=1 / 2$ from Eq. (64) and $v_{24}^{v d}=v_{24}^{v}+v_{24}^{d}$. In addition, based also on Eq. (9), we have written $v_{\mathbf{B C}}=\hat{w}_{3} 2 v_{34}=2 \nu_{34}$ and $v_{\mathbf{B A}}=\hat{w}_{3} 2 \nu_{32}=2 v_{32}$, since $\hat{w}_{3}=1$ from Eq. (64). Finally, the rate for path $\mathbf{A B C}$ is divided by 2 in order to avoid counting the hops within the path twice when considering the complementary unit cell (see Sec. II B 10). This is equivalent to dividing every rate within the path by 2 .

Similarly, the hop rate from plane $\mathbf{C}$ to plane $\mathbf{A}^{\prime}$ is

$$
\begin{aligned}
v_{\mathbf{C} \mathbf{A}^{\prime}}^{+} & =v_{\mathbf{C} \mathbf{A}^{\prime}}+\frac{1}{2} v_{\mathbf{C D A}} \\
& =v_{\mathbf{C} \mathbf{A}^{\prime}}+\frac{1}{2} \frac{v_{\mathbf{C D}} v_{\mathbf{D A}}}{v_{\mathbf{D C}}+v_{\mathbf{D A}^{\prime}}} \\
& =\hat{w}_{4 a} v_{42}^{v d}+\hat{w}_{4 b} v_{42}^{v d}+\frac{1}{2} \frac{\left(\hat{w}_{4 a} v_{41}+\hat{w}_{4 b} v_{41}\right) \hat{w}_{1} 2 v_{12}}{\hat{w}_{1} 2 v_{14}+\hat{w}_{1} 2 v_{12}} \\
& =\frac{2 v_{42}^{v d}\left(v_{12}+v_{14}\right)+v_{41} v_{12}}{2\left(v_{12}+v_{14}\right)}
\end{aligned}
$$

where we have used Eq. (91) to describe the rate for path $\mathbf{C D A}^{\prime}$ $\left[v_{\mathbf{C D A}^{\prime}}=v_{\mathbf{C D}} v_{\mathbf{D A}^{\prime}} /\left(v_{\mathbf{D C}}+v_{\mathbf{D A}^{\prime}}\right)\right], \hat{w}_{4 a}=\hat{w}_{4 b}=1 / 2$ and $\hat{w}_{1}=$ 1 from Eq. (64), and $v_{42}^{v d}=v_{42}^{v}+v_{42}^{d}$. Finally, the rate to hop from plane $\mathbf{C}$ to plane $\mathbf{A}$ becomes

$$
\begin{aligned}
v_{\mathbf{C A}}^{-} & =v_{\mathbf{C A}}+\frac{1}{2} v_{\mathbf{C B A}} \\
& =v_{\mathbf{C A}}+\frac{1}{2} \frac{v_{\mathbf{C B}} v_{\mathbf{B A}}}{v_{\mathbf{B C}}+v_{\mathbf{B A}}} \\
& =\hat{w}_{4 a} v_{42}^{v d}+\hat{w}_{4 b} v_{42}^{v d}+\frac{1}{2} \frac{\left(\hat{w}_{4 a} v_{43}+\hat{w}_{4 b} v_{43}\right) \hat{w}_{3} 2 v_{32}}{\hat{w}_{3} 2 v_{32}+\hat{w}_{3} 2 v_{34}} \\
& =\frac{2 v_{42}^{v d}\left(v_{32}+v_{34}\right)+v_{43} v_{32}}{2\left(v_{32}+v_{34}\right)}
\end{aligned}
$$


Using Eq. (91) and Eqs. (122), (126), and (130), the effective hop rate across the primary cell becomes

$$
\begin{aligned}
v_{\mathbf{A} \mathbf{A}, y}^{*} & =\frac{v_{\mathbf{A C}^{+}}^{+} v_{\mathbf{C A}^{\prime}}^{+}}{v_{\mathbf{C A}}^{-}+v_{\mathbf{C A}^{\prime}}^{+}} \\
& =\frac{1}{2} \frac{\left[2 v_{24}^{v d}\left(v_{32}+v_{34}\right)+v_{23} v_{34}\right]\left[2 v_{42}^{v d}\left(v_{12}+v_{14}\right)+v_{41} v_{12}\right]}{\left[2 v_{42}^{v d}\left(v_{32}+v_{34}\right)+v_{43} v_{32}\right]\left(v_{12}+v_{14}\right)+\left[2 v_{42}^{v d}\left(v_{12}+v_{14}\right)+v_{41} v_{12}\right]\left(v_{32}+v_{34}\right)} .
\end{aligned}
$$

This is the same as Eq. (69) in Sec. II F. Now, considering the complementary unit cell [Fig. 2(m)], we have

$$
\begin{aligned}
v_{\mathbf{D D}, y}^{*} & =\frac{v_{\mathbf{D B}}^{+} v_{\mathbf{B D}^{\prime}}^{+}}{v_{\mathbf{B D}}^{-}+v_{\mathbf{B D}^{\prime}}^{+}} \\
& =\frac{\left[v_{13}\left(v_{21}+v_{23}\right)+v_{12} v_{23}\right]\left[v_{31}\left(v_{41}+v_{43}\right)+v_{34} v_{41}\right]}{\left[v_{31}\left(v_{21}+v_{23}\right)+v_{32} v_{21}\right]\left(v_{41}+v_{43}\right)+\left[v_{31}\left(v_{41}+v_{43}\right)+v_{34} v_{41}\right]\left(v_{21}+v_{23}\right)},
\end{aligned}
$$

where we have used

$$
\begin{aligned}
v_{\mathbf{D B}}^{+} & =v_{\mathbf{D B}}+\frac{1}{2} v_{\mathbf{D A B}}=v_{\mathbf{D B}}+\frac{1}{2} \frac{v_{\mathbf{D A}} v_{\mathbf{A B}}}{v_{\mathbf{A D}}+v_{\mathbf{A B}}} \\
& =\hat{w}_{1} v_{13}+\frac{1}{2} \frac{\hat{w}_{1} 2 v_{12}\left(\hat{w}_{2 a} v_{23}+\hat{w}_{2 b} v_{23}\right)}{\hat{w}_{2 a} v_{21}+\hat{w}_{2 b} v_{21}+\hat{w}_{2 a} v_{23}+\hat{w}_{2 b} v_{23}} \\
& =\frac{v_{13}\left(v_{21}+v_{23}\right)+v_{12} v_{23}}{v_{21}+v_{23}} \\
v_{\mathbf{B D}^{\prime}}^{+} & =v_{\mathbf{B D}^{\prime}}+\frac{1}{2} v_{\mathbf{B C D}}=v_{\mathbf{B D}}+\frac{1}{2} \frac{v_{\mathbf{B C}} v_{\mathbf{C D}^{\prime}}}{v_{\mathbf{C B}}+v_{\mathbf{C D}^{\prime}}} \\
& =\hat{w}_{3} v_{31}+\frac{1}{2} \frac{\hat{w}_{3} 2 v_{34}\left(\hat{w}_{4 a} v_{41}+\hat{w}_{4 b} v_{41}\right)}{\hat{w}_{4 a} v_{43}+\hat{w}_{4 b} v_{43}+\hat{w}_{4 a} v_{41}+\hat{w}_{4 b} v_{41}} \\
& =\frac{v_{31}\left(v_{41}+v_{43}\right)+v_{34} v_{41}}{v_{41}+v_{43}} \\
v_{\mathbf{B D}}^{-} & =v_{\mathbf{B D}}+\frac{1}{2} v_{\mathbf{B A D}}=v_{\mathbf{B D}}+\frac{1}{2} \frac{v_{\mathbf{B A}} v_{\mathbf{A D}}}{v_{\mathbf{A B}}+v_{\mathbf{A D}}} \\
& =\hat{w}_{3} v_{31}+\frac{1}{2} \frac{\hat{w}_{3} 2 v_{32}\left(\hat{w}_{2 a} v_{21}+\hat{w}_{2 b} v_{21}\right)}{\hat{w}_{2 a} v_{23}+\hat{w}_{2 b} v_{23}+\hat{w}_{2 a} v_{21}+\hat{w}_{2 b} v_{21}} \\
& =\frac{v_{31}\left(v_{21}+v_{23}\right)+v_{32} v_{21}}{v_{21}+v_{23}} .
\end{aligned}
$$

Equation (134) is identical to Eq. (75). Thus, the diffusivity is given by Eq. (76). Altogether, the previous derivations show that the 1D formulas obtained in Sec. III provide a fast and easy procedure for calculating the diffusivity.

\section{Other examples}

Following the procedure presented in Secs. IV A and IV B, Appendix 2 in the Supplemental Material [14] shows that the components of the diffusivity for the systems displayed in Fig. 3 are as follows.

Figures 3(a)-3(d):

$D_{T, x}^{\theta \approx 0}=\frac{\left(2 v_{21}^{d}+v_{21}^{v}\right)\left(v_{11}+v_{12}^{d}\right)+\left(2 v_{12}^{d}+v_{12}^{v}\right) v_{21}^{d}}{4 v_{12}^{d}+2 v_{12}^{v}+2 v_{21}^{d}+v_{21}^{v}} 4 a_{o}^{2}$,

$D_{T, y}^{\theta \approx 0}=\frac{\left(2 v_{21}^{d}+v_{21}^{v}\right)\left[v_{11}\left(2 v_{22}+2 v_{21}^{d}+v_{21}^{v}\right)+2 v_{12}^{v}\left(v_{22}+v_{21}^{d}\right)\right]}{\left(4 v_{12}^{d}+2 v_{12}^{v}+2 v_{21}^{d}+v_{21}^{v}\right)\left(2 v_{22}+2 v_{21}^{d}+v_{21}^{v}\right)} 4 a_{o}^{2}$.
Figures 3(e)-3(h):

$$
\begin{aligned}
D_{T, x}^{\theta \approx 0} & =\frac{9 v_{21} v_{13} v_{31}\left(v_{11} v_{22}+v_{11} v_{21}+v_{12} v_{22}\right)}{4 B\left(v_{11} v_{22}+v_{11} v_{21}+v_{12} v_{22}+v_{13} v_{22}+v_{13} v_{21}\right)} a_{o}^{2}, \\
D_{T, y}^{\theta \approx 0} & =\frac{v_{21}\left[v_{12} v_{31}^{2}+\left(2 v_{12}+v_{13}\right) v_{33} v_{31}+v_{13} v_{33}^{2}\right]}{B\left(2 v_{33}+v_{31}\right)} a_{o}^{2} .
\end{aligned}
$$

Above, $B=v_{21} v_{31}+v_{31} v_{12}+v_{21} v_{13}$. Similarly, Appendix 3 in the Supplemental Material [14] shows that the components of the diffusivity for the systems displayed in Fig. 1 are as follows.

Figure 1(a):

$$
\begin{aligned}
D_{T, x}^{\theta \approx 0} & =\frac{4 v_{12} \nu_{23} \nu_{32} \nu_{21}}{\left(v_{21}+2 v_{23}\right)\left(\nu_{32} \nu_{21}+2 v_{32} v_{12}+2 v_{12} \nu_{23}\right)} l^{2}, \\
D_{T, y}^{\theta \approx 0} & =\frac{4 v_{12} v_{23} \nu_{32}}{v_{32} \nu_{21}+2 v_{32} v_{12}+2 v_{12} \nu_{23}} l^{2} .
\end{aligned}
$$

Figure 1(b):

$$
\begin{aligned}
& D_{T, x}^{\theta \approx 0}=\frac{4 v_{12} v_{21}\left(v_{22}^{h}+2 v_{22}^{d}\right)}{\left(2 v_{12}+v_{21}\right)\left(v_{21}+2 v_{22}^{h}+4 v_{22}^{d}\right)} a^{2}, \\
& D_{T, y}^{\theta \approx 0}=\frac{v_{21} v_{11}+2 v_{12}\left(v_{22}^{v}+v_{22}^{d}\right)}{2 v_{12}+v_{21}} a^{2} .
\end{aligned}
$$

Figure 1(c):

$$
\begin{aligned}
D_{T, x}^{\theta \approx 0} & =\frac{v_{31} v_{11}^{l}\left(v_{11}^{L}+v_{13}\right)}{\left(v_{13}+2 v_{31}\right)\left(v_{11}^{l}+v_{11}^{L}+v_{13}\right)}(l+L)^{2}, \\
D_{T, y}^{\theta \approx 0} & =\frac{v_{13} \nu_{31}}{v_{13}+2 v_{31}} a^{2} .
\end{aligned}
$$

Figure 1(d):

$$
\begin{aligned}
& D_{T, x}^{\theta \approx 0}=\frac{v_{12}^{a b c d}}{v_{12}^{a b c d}+v_{21}^{a b c d}} \frac{v_{21}^{a} v_{12}^{b}}{v_{12}^{a}+v_{12}^{b}}(a+b)^{2}, \\
& D_{T, y}^{\theta \approx 0}=\frac{v_{12}^{a b c d}}{v_{12}^{a b c d}+v_{21}^{a b c d}} \frac{v_{21}^{c} v_{12}^{d}}{v_{12}^{c}+v_{12}^{d}}(c+d)^{2} .
\end{aligned}
$$

In addition, Appendix 4 and Fig. 5 in the Supplemental Material [14] show that the components of the diffusivity for the corresponding systems are as follows. 
TABLE II. Procedure M-2 to determine the effective hop rates $v_{\mathbf{T T}, l}^{*}$ and $\nu_{\mathbf{X} \mathbf{x}, l}^{*}$.

Determine $w_{i}$ [site occupancies, Eq. (3)].

Define an orthorhombic unit cell.

(1) For every dimension $l(X, Y$, and $Z)$ :

Relabel all equivalent sites. Determine $w_{i}^{*}$ [effective occupancies of the relabeled sites, Eq. (6)].

Define the planes $\mathbf{A}, \mathbf{B}, \mathbf{C}, \ldots, \mathbf{X}, \mathbf{A}^{\prime}$.

Determine $w_{\mathbf{P}}^{*}$ [effective occupancies of planes, Eq. (7)].

Determine $\hat{w}_{i}$ [in-plane occupancies, Eq. (8)].

Determine $\nu_{\mathbf{P Q}}$ [hop rates between planes, Eq. (9)].

Consider the $1 \mathrm{D}$ representation. If boundary crossings occur, find the lower complementary cell (with the lower terminal at $\mathbf{X}$ ). Distribute the hops between primary and complementary cells following rules R1-R3 (Sec. II B 10).

(2) For every unit cell (primary/complementary):

Consider the system as a 1D path. If long hops with hop-commensurate paths occur between two planes, the effective hop rate between those planes is the sum of the rates of the long hop and the hop-commensurate path.

Determine the effective hop rate for $S$ distinct planes [ $v_{\mathbf{T T}, l}^{*}$, Eq. (93)]; Eq. (90) can be used for $S=4$, and Eqs. (91) and (92) can be used for $S=2$ and $S=3$, respectively. (2) End. (For the complementary cell this directly gives $v_{\mathbf{X X}, l}^{*}$.)

(1) End.

Figure 5(a):

$$
\begin{aligned}
D_{T, x}^{\theta \approx 0} & =\frac{\Gamma}{\Gamma+\Gamma_{1}(n-1)} \frac{\Gamma_{1} S}{1+(n-1) S}(n a)^{2}, \\
D_{T, y}^{\theta \approx 0} & =\frac{\Gamma\left[\Gamma_{1}(n-1)+\Gamma_{2}\right]}{\Gamma+\Gamma_{1}(n-1)} a^{2} .
\end{aligned}
$$

Figure 5(b):

$$
\begin{aligned}
& D_{T, x}^{\theta \approx 0}=\left(4 \widetilde{v}_{11}+v_{11}\right) 4 a_{o}^{2}, \\
& D_{T, y}^{\theta \approx 0}=v_{11} 4 a_{o}^{2} .
\end{aligned}
$$

Figure 5(c):

$$
\begin{aligned}
D_{T, x}^{\theta \approx 0} & =\frac{9 v_{12} v_{21}}{2\left(v_{12}+v_{21}\right)} a_{o}^{2}, \\
D_{T, y}^{\theta \approx 0} & =\frac{v_{12} v_{22}+v_{21} v_{11}+v_{12} v_{21}}{2\left(v_{12}+v_{21}\right)} a_{o}^{2} .
\end{aligned}
$$

\section{Algorithm (M-2)}

The procedure presented in Secs. IV A and IV B can be summarized as the algorithm reported in Table II. The procedure is referred to as M-2 to differentiate it from the method described in Sec. II G. In practice, both formulations (M-1 and M-2) can be used to analyze the different contributions to the low coverage diffusivity. Although M-1 can be automated easily, it may require lengthy calculations to compute the determinants $B^{*}$ and $B_{\mathbf{P}, \mathbf{T} \square}^{*}\left(\right.$ or $B_{\mathbf{P}, \square \mathbf{T}^{\prime}}^{*}$ ). In comparison, M-2 provides a more immediate route, at the cost of analyzing further how the $1 \mathrm{D}$ representation of the primary/complementary cell should be split into sections so that the hop rates of the long hops and their commensurate paths are summed together. Note, however, that the two procedures are completely equivalent.

In addition to the effective hop rates derived for the systems in Figs. 1-5, Table 3 in the Supplemental Material [14] provides the final expressions obtained for the low coverage tracer diffusivity for a triangular lattice with four types of adsorption sites and asymmetric hops. Furthermore, we stress that all the expressions listed in Tables PI-I, PI-III, and PI-IV for systems with symmetric hops only (Part I [1]) have also been derived using methods M-1 and M-2 in this report (Part II). This confirms that the most general formula described in the present report [Eq. (23)] is a suitable generalization of Eq. (2).

\section{VALIDATION}

\section{A. Comparison to simulations}

Here we verify the validity of the proposed diffusivity formulas against kinetic Monte Carlo simulations. The details of the simulations are given in Sec. PI-III (Part I [1]). Table IV in the Supplemental Material [14] provides a comparison between the simulations and the theory for various systems with asymmetric hops. Since the goal is to check the validity of the analytical formulas, different hop rate values are used to probe situations where the rates have similar values or differ by several orders of magnitude. In fact, realistic hop rates are used for the systems in Figs. 1(a)-1(c) and 2, where the activation energies are taken from previous DFT studies, as indicated in the corresponding figure captions. The relative values of the removal rates are strongly modified by varying the temperature. Near room temperature $(300 \mathrm{~K})$, only a few hop types are active (the rest of the hops have very low rates by orders of magnitude). On the contrary, at high temperatures (such as $1500 \mathrm{~K}$, as an example) all different hop rates become rather similar. Overall, we stress that the analytical formulas derived in both Part I and Part II of this study compare extremely well with the simulations over diffusivity values that span many orders of magnitude. This strongly indicates that the proposed formalism is exact. Therefore, it can be concluded that the proposed formalism is suitable for study of the low coverage tracer diffusivity of typical diffusion species in complex, periodic energy landscapes without any need to perform the corresponding KMC simulations.

\section{B. Comparison to previous studies}

Previously, tracer diffusion has been studied using the continuous-time random walk formalism [2,3]. While a traditional random walk considers only a single hop type, the CTRW method contemplates the possibility of different hop rates for different hops. As in the present study, the CTRW formalism considers the corresponding network of jumps between all the different sites and solves the master equation for the conditional probabilities of the hops in the long-time limit. For this purpose, however, Fourier and Laplace transformations are applied to the master equation in the CTRW formalism and the resulting matrix equation (in reciprocal space or $k$ space) is diagonalized (eigenvalue decomposition). The diffusivity is found by considering the 
longest-living mode, i.e., the eigenvalue that vanishes at $k=0$ (hydrodynamic limit).

The CTRW method [2,3] has been used to study adatom diffusion in various systems, including (A) a generic stepped surface [13], (B) Ga diffusion on $\mathrm{GaAs}(001)-(\beta \times 4)$ [7], (C) diffusion on $\operatorname{GaAs}(001)-\mathrm{c}(4 \times 4)$ [9], and (D) diffusion on $\mathrm{In}_{2 / 3} \mathrm{Ga}_{1 / 3} \mathrm{As}(001)-(2 \times 3)$ and $\mathrm{In}_{2 / 3} \mathrm{Ga}_{1 / 3} \mathrm{As}(001)-(1 \times 3)$ [10]. As shown below, the formalism proposed in the present study corrects some of the expressions obtained in those studies and provides new formulas for several cases that could not be solved by the CTRW approach.

Regarding system (A), which is shown in Fig. 5(a) in the Supplemental Material [14], the expressions we obtain for the Cartesian components of the diffusivity [Eq. (150)] are the same as those reported by Natori and Godby [13]. However, the analytical derivation based on our formalism (see Appendix 4(a) in the Supplemental Material [14]) is significantly shorter than that in the original paper.

Regarding the diffusivity in system (B), Kley et al. [7] study two hopping networks, here referred to as case 1 [no boundary crossings; see Fig. 1(b)] and case 2 (with boundary crossings; see Fig. 2). Using the names for the hop rates introduced in the present study, the expressions that Kley et al. [7] provide for the two components of the diffusivity for case 1 read

$$
\begin{aligned}
& D_{T, x,(K)}^{\theta \approx 0}=\frac{8 v_{12} v_{21}\left(v_{22}^{h}+2 v_{22}^{d}\right)}{\left(2 v_{12}+v_{21}\right)\left(v_{21}+2 v_{22}^{h}+4 v_{22}^{d}\right)} a_{o}^{2}, \\
& D_{T, y,(K)}^{\theta \approx 0}=\frac{2\left(v_{21} v_{11}+4 v_{12} v_{22}^{d}\right)}{2 v_{12}+v_{21}} a_{o}^{2} .
\end{aligned}
$$

Equation (153) is similar (but not identical) to our result [Eq. (147)]. For Kley et $a l ., a_{o}$ in Eq. (153) is the lattice constant of the conventional unit cell of the zincblende structure $(5.65 \AA$ for GaAs). However, in the present study $a$ in Eq. (147) is the lattice constant of the surface unit cell along the [110] direction [7.99 $\AA$ for $\operatorname{GaAs}(001)]$. Since $a=\sqrt{2} a_{o}$, the two expressions for the $X$ component become identical. However, the $Y$ component of Kley et al. can only be reconciled with our formula by taking $v_{22}^{v}=v_{22}^{d}$. Although it is likely that these two hops have the same activation barrier and, thus, their rates are equal, our expression for the $Y$ component is more general.

Now, regarding case 2, Kley et al. make the following observation: "With the CTRW formalism we approximate the diffusion constants (the rather long exact expression will be published elsewhere [28]) as"

$$
\begin{aligned}
& D_{T, x,(K)}^{\theta \approx 0} \approx \frac{8\left(v_{12} v_{31}+v_{13} v_{32}\right)}{2 v_{13}} a^{2}, \\
& D_{T, y,(K)}^{\theta \approx 0} \approx \frac{2 v_{11} v_{31}}{v_{13}} a^{2} .
\end{aligned}
$$

Unfortunately, we have not been able to find the exact expression in subsequent publications by Kley et al. [Note that Ref. [28] in the original article reads "[28] A. Kley (unpublished)"]. According to our formalism, the exact expressions for the diffusivity are shown in Eqs. (62) and (76) for the $X$ and $Y$ components, respectively. This system features boundary crossings in the $Y$ dimension markedly and it is possible that previous efforts have been unable to solve the problem correctly. In comparison, the origin of the complex expression for the $Y$ component of the diffusivity is simple to understand within our formalism. The fact that the diffusivity is the same for different choices of the primary and complementary unit cells strongly validates our approach. In addition, the excellent comparison to the numerical simulations confirms the validity of our method (see Sec. V A).

Regarding the diffusivity for system (C), also Penev et al. [9] study two hopping networks, namely, case 3 , for high temperature without boundary crossings (see Fig. 5(b) in the Supplemental Material [14]), and case 4, for low temperature with boundary crossings [see Figs. 3(a)-3(d)]. For case 3, the expressions we obtain for the Cartesian components of the diffusivity [Eq. (151)] are the same as those reported by Penev et al. [9]. In turn, for case 4, Penev et al. make the following observation: "At sufficiently low temperatures, however, one should include A2 in the 2D network of sites and consider branching of the diffusion pathways towards neighboring A1 or A2 sites. Although an analytic result for $D_{\alpha \beta}$ can still be derived in this case within the CTRW formalism, the expressions are rather cumbersome and one has to seek for simplifications requiring knowledge of all $\Gamma_{f i}$ rates." In comparison, the exact expressions for the Cartesian components of the diffusivity derived within our formalism are reasonably simple, as shown in Eq. (144). As before, these formulas remain unchanged when using different primary and/or complementary unit cells, and their agreement with the KMC simulations is excellent (see Sec. V A).

A related system was studied by Lepage et al. [6], who considered the same substrate, $\operatorname{GaAs}(001)-\mathrm{c}(4 \times 4)$, with a different adatom $(\mathrm{Ga})$. The potential energy surface for $\mathrm{Ga}$ is more corrugated, having deeper minima/higher barriers and additional adsorption sites compared to the In adatom considered by Penev et al. The network of hops for $\mathrm{Ga}$ is presented in Fig. 1(a). Although Lepage et al. did not attempt to obtain any analytical expression for the diffusivity, their KMC simulations agree well with ours. In turn, our simulations show excellent agreement with the analytical expressions derived within our formalism [Eq. (146)].

Regarding the diffusivity for system (D) with $(1 \times 3)$ reconstruction (case 6; see Fig. 5(c) in the Supplemental Material [14]), the expressions we obtain for the Cartesian components of the diffusivity [Eq. (152)] are the same as those reported by Penev et al. [10]. When comparing their equations to ours one should consider that they write $\Gamma_{f i}$ where we use $v_{i f}$. In turn, for system (D) with $(2 \times 3)$ reconstruction [case 5; see Figs. 3(e)-3(h)], we obtain the same formula for the $X$ component of the diffusivity. Using the terms for the hop rates introduced in the present study, the expression that Penev et al. [10] give for the $Y$ component of the diffusivity is

$$
D_{T, y}^{\theta \approx 0}=\frac{v_{21}\left[v_{12} v_{31}^{2}+\left(2 v_{12}+v_{13}\right) v_{33} v_{31}+3 v_{13} v_{33}^{2}\right]}{B\left(2 v_{33}+v_{31}\right)} a_{o}^{2} .
$$

Equation (155) is similar (but not identical) to our result [Eq. (145)]. They differ in the factor 3 that multiplies the term $v_{13} v_{33}^{2}$ in Penev et al.'s expression. The correct value of this factor is 1, as in Eq. (145). This is supported by the fact that we can derive Eq. (145) using different unit cells, all giving the same result. In addition, poor agreement with the 
kinetic Monte Carlo simulations is obtained if the value 3 is used instead of 1.

In summary, the proposed method in the present study agrees well with previous analytical results obtained using the CTRW method, correcting some of them and even providing new formulas for difficult cases that could not be solved before. In general, the expressions obtained using the CTRW method are approximate in the sense that only the leading terms of an expansion are kept. This may explain why some CTRW results differ from the exact expressions derived here using the proposed new method.

\section{Independent and coupled diffusion channels}

A major difference with respect to the CTRW method is the direct use of real space in the formalism proposed in the present study. This makes possible the identification of different diffusion channels in complex systems. For instance, although we can only distinguish one diffusion channel in the $1 \mathrm{D}$ representation shown in Fig. 2(f) for propagation along $X$, the 1D representations shown in Figs. 2(l) and 2(m) for propagation along $Y$ suggest the existence of two independent channels $\left(\mathbf{A C A}^{\prime}, \mathbf{D B D} \mathbf{D}^{\prime}\right)$ that are coupled through a third one (ABCDA'). We may close any of the three channels (or even two of them) and the adparticle will still be propagated. Since every channel is composed of sections, we may close one or several sections without completely canceling the diffusivity. In order to neutralize the diffusivity completely we need to close the three channels. Other examples are shown in Figs. 3(b) and 3(c), where three independent diffusion channels are observed, namely, $\mathbf{A D A} \mathbf{A}^{\prime}, \mathbf{A C D E} \mathbf{A}^{\prime}$, and $\mathbf{F A B D F}{ }^{\prime}$, and in Figs. 3(f) and 3(g), with two independent channels (ACA $\mathbf{A}^{\prime}$ and $\left.\mathbf{D A B D}^{\prime}\right)$. From this perspective, the complex system reported in Table III(c) (see the Supplemental Material [14]) has a total of five independent diffusion channels, leading to the five diffusivity terms $w_{f} 3 v_{f f}, w_{f} v_{f t}, w_{h} 3 v_{h h}, w_{h} v_{h t}$, and $\frac{w_{b}}{3} v_{o}$.

The real-space analysis performed in this study concludes that complex tracer diffusion in periodic energy landscapes in any number of dimensions can be reduced to a $1 \mathrm{D}$ problem of hopping between planes. The previous examples show that the number and structure of the different diffusion channels are revealed once the $1 \mathrm{D}$ picture of the system is constructed. This requires observing rules R1-R3 (Sec. II B 10), if boundary crossings occur. In comparison, the CTRW method does not provide direct access to the structure of the diffusion channels, and depending on the complexity of the system, it may only provide an approximation of the actual tracer diffusivity.

\section{CONCLUSIONS}

This study completes a series of two papers focusing on the presentation of a general expression for calculation of the single-particle diffusion coefficient in any number of dimensions for systems with complex, periodic energy landscapes, where the end sites of the hops can be located symmetrically (Part I) and/or asymmetrically (Part II) with respect to the hop origins. To use these expressions, the only necessary input are the values of the hop rates, which can be determined from the corresponding diffusion barriers by experiments and/or calcu- lations based on DFT. This provides a useful alternative to the traditional, labor-intensive procedure where numerous kinetic Monte Carlo simulations must be performed after knowledge of the diffusion barriers. Furthermore, the proposed formalism is an alternative to the traditional continuous-time random walk formalism. Compared to the analysis of diffusion performed in reciprocal space by the CTRW formalism, the proposed method is more intuitive, directly using the information on the hop rates in real space. Furthermore, for very complex systems the proposed, exact formalism enables the identification of the underlying (independent and/or coupled) diffusion channels.

The present report (Part II) describes the low coverage diffusivity $\left(D_{T}^{\theta \approx 0}\right)$ as the mean of the Cartesian components $\left(D_{T, x}^{\theta \approx 0}, \quad D_{T, y}^{\theta \approx 0}, \ldots\right): D_{T}^{\theta \approx 0}=\frac{1}{\alpha} \Sigma_{l=1}^{\alpha} D_{T, x_{l}}^{\theta \approx 0}$, where $\alpha$ is the number of dimensions and $x_{1}=x, x_{2}=y, x_{3}=z$ designate the actual dimensions [Eq. (18)]. In turn, the Cartesian component for dimension $l(=1, \ldots, \alpha)$ is described as a weighted sum of the partial diffusivities for the cellcommensurate sites $(i \in \mathcal{C})$, terminal sites $(i \in \mathcal{T})$, and crosser sites $(i \in \mathcal{X}): D_{T, x_{l}}^{\theta \approx 0}=\Sigma_{i \in \mathcal{C} \cup \mathcal{T} \cup \mathcal{X}} w_{i}^{*} v_{i i, l}^{*} a_{l}^{2}=\Sigma_{i \in \mathcal{C}} w_{i} v_{i i, l} a_{l}^{2}+$ $\Sigma_{i \in \mathcal{T}} w_{i}^{*} v_{i i, l}^{*} a_{l}^{2}+\Sigma_{i \in \mathcal{X}} w_{i}^{*} v_{i i, l}^{*} a_{l}^{2}$ [Eq. (21)]. Here, $a_{l}$ is the lattice parameter (along dimension $l$ ) for the chosen orthorhombic unit cell and the occupancies, $w_{i}$, are determined from the rateplicities [Eq. (3)]: $w_{i}=\frac{\mu_{j i}}{\mu_{i j}} / \Sigma_{k} \frac{\mu_{j k}}{\mu_{k j}}$, where the rateplicity $\mu_{i j}$ is the product of the hop rate from site $i$ to site $j, v_{i j}$, and the number of equivalent hops from $i$ to $j$, i.e., the multiplicity, $m_{i j}$. [The effective occupancies, $w_{i}^{*}=w_{i} / n_{i}$, are directly obtained by dividing the occupancies by the number of times that site $i$ appears in the orthorhombic unit cell, $\left.n_{i}\right]$. Since the hop rates for the cell-commensurate hops are known, the diffusivity can be calculated once the effective hop rates for the terminal and crosser sites, $v_{i i, l}^{*}$, have been determined, for which purpose the present study (Part II) provides two complementary methods (M-1, in Sec. II G, and $\mathrm{M}-2$, in Sec. IV D).

Based on the use of Eqs. (39) and (40), method M-1 can be automated easily, but it requires longer calculations. In comparison, $\mathrm{M}-2$ is based on the $1 \mathrm{D}$ formulas summarized in Eqs. (90)-(92), which provide a more direct approach to determining the effective hop rates. However, this requires analyzing how the $1 \mathrm{D}$ representation of the primary/complementary cell should be split into sections so that the hop rates of the long hops and their commensurate paths are summed together.

The central result of the proposed formalism [Eq. (23)] is used to derive numerous formulas for a variety of systems, which are then validated against KMC simulations and previous analytical expressions based on the CTRW formalism. The proposed method corrects some of the formulas obtained by the CTRW method and provides new expressions for previously unsolved cases, thus demonstrating the ability of the proposed formalism to describe diffusion.

The proposed formalism may have implications for the description of transport in other areas based on hopping theories that are solved in reciprocal space. A description in real space might be possible for such systems by defining the corresponding site occupancies and effective occupancies for the various planes, thus assigning hopping rates to the planes, as illustrated in this study. 


\section{ACKNOWLEDGMENTS}

We acknowledge support from the Ramón y Cajal Fellowship Program of the Spanish Ministry of Science and Innovation (M. A. Gosálvez), the JAE-Doc grant from the 'Junta para la Ampliación de Estudios' program cofunded by FSE (N. Ferrando), the University of the Basque Country (Grant No. GIC07IT36607), the Spanish Ministry of Science and Innovation (Grants No. FIS2013-48286-C02-02-P and No. FIS2013-48286-C02-01-P), the Basque Government through the Nanomaterials project under the nanoGUNE2014 program (Grant No. IE05-151), the Tomsk State University Academic D. I. Mendeleev Fund Program in 2015 (Research Grant No. 8.1.05.2015), and partial support from Saint Petersburg State University (Project No. 15.61.202.2015).
[1] M. A. Gosálvez, M. M. Otrokov, N. Ferrando, A. G. Ryabishchenkova, A. Ayuela, P. M. Echenique, and E. V. Chulkov, Phys. Rev. B 93, 075429 (2016).

[2] J. W. Haus and K. W. Kehr, Phys. Rep. 150, 263 (1987).

[3] R. Gomer, Rep. Prog. Phys. 53, 917 (1990).

[4] Y. Xia, D. Qian, D. Hsieh, L. Wray, A. Pal, H. Lin, A. Bansil, D. Grauer, Y. S. Hor, R. J. Cava, and M. Z. Hasan, Nat. Phys. 5, 398 (2009).

[5] S. Roy, H. L. Meyerheim, K. Mohseni, A. Ernst, M. M. Otrokov, M. G. Vergniory, G. Mussler, J. Kampmeier, D. Grützmacher, C. Tusche, J. Schneider, E. V. Chulkov, and J. Kirschner, Phys. Rev. B 90, 155456 (2014).

[6] J. G. LePage, M. Alouani, D. L. Dorsey, J. W. Wilkins, and P. E. Blöchl, Phys. Rev. B 58, 1499 (1998).

[7] A. Kley, P. Ruggerone, and M. Scheffler, Phys. Rev. Lett. 79, 5278 (1997).
[8] S. M. Lee, S.-H. Lee, and M. Scheffler, Phys. Rev. B 69, 125317 (2004).

[9] E. Penev, P. Kratzer, and M. Scheffler, Phys. Rev. B 64, 085401 (2001).

[10] E. Penev, S. Stojković, P. Kratzer, and M. Scheffler, Phys. Rev. B 69, 115335 (2004).

[11] R. Condit, in Kinetics of Reactions in Ionic Systems, Materials Science Research, edited by T. Gray and V. Fréchette (Springer, New York, 1969), Chap. 15.

[12] D. Birnie, J. Phys. Chem. Solids 51, 1313 (1990).

[13] A. Natori and R. W. Godby, Phys. Rev. B 47, 15816 (1993).

[14] See Supplemental Material at http://link.aps.org/supplemental/ 10.1103/PhysRevB.93.205416 for Appendixes 1-4 and additional figures. 